\title{
Información bioantropológica publicada de Patagonia Austral. Un abordaje de la situación actual desde el data-sharing
}

(跑) RMA

Published bioanthropological information from Patagonia Austral. An approach from current tendency of data sharing

\author{
Manuel D. D’Angelo del Campo*, Pamela García Laborde ${ }^{* * 1}$, \\ Luciano O. Valenzuela ${ }^{\star * 2}$, Josefina M. B. Motti**3, Marilina \\ Martucci**4, Patricia I. Palacio ${ }^{* * * 1}$ y Ricardo A. Guichón ${ }^{* * 2}$
}

Antropología Biológica

*Laboratorio de Ecología Evolutiva Humana (LEEH), Núcleo de Estudios Interdisciplinarios de Poblaciones Humanas de Patagonia Austral (NEIPHPA), Facultad de Ciencias Sociales (FACSO), Universidad del Centro de

la Provincia de Buenos Aires (UNCPBA). Calle 508 Nº 881. CP: 7631. Quequén, Bs. As. Argentina. Laboratorio de Poblaciones de Pasado (LAPP), Departamento de Biología, Facultad de Ciencias, Universidad Autónoma de Madrid (UAM), C/Darwin 2, E-28049, Madrid, España. manueldomingodangelo@gmail.com

**Laboratorio de Ecología Evolutiva Humana (LEEH), Núcleo de Estudios Interdisciplinarios de Poblaciones Humanas de Patagonia Austral (NEIPHPA), Facultad de Ciencias Sociales (FACSO), Universidad del Centro de la Provincia de Buenos Aires (UNCPBA). CONICET. Calle 508 N 881. CP: 7631. Quequén, Bs. As. Argentina. PPamelagl1981@hotmail.com 2lucianoovalenzuela@gmail.com 3josemotti@yahoo.com.ar 4lucianoovalenzuela@gmail.com

***Laboratorio de Ecología Evolutiva Humana (LEEH), Núcleo de Estudios Interdisciplinarios de Poblaciones Humanas de Patagonia Austral (NEIPHPA), Facultad de Ciencias Sociales (FACSO), Universidad del Centro de la Provincia de Buenos Aires (UNCPBA). Calle 508 Nº 881. CP: 7631. Quequén, Bs. As. Argentina. Departamento de Biología, Facultad de Ciencias Exactas y Naturales, Universidad Nacional de Mar del Plata. 1patriciapalacio@gmail.com ${ }^{2}$ ricardoguichon@hotmail.com

\begin{abstract}
Resumen
Los avances técnicos de las últimas décadas han incidido en el ámbito científico conllevando un aumento en la generación de nuevos conocimientos. Estos nuevos desarrollos han permitido mejorar las comunicaciones y el acceso a la información. En estas condiciones, aparece una corriente global, el data sharing, que aboga por la libre puesta en disposición de los datos producto de las investigaciones científicas. Esta tendencia no está desarrollada en el área de Patagonia Austral. Sin embargo, antes de intercambiar datos, es importante conocer qué tipo de información se puede o pretende compartir. Con el fin de acercarnos a la situación en la que se encuentra la información disponible en la región, se realizó un análisis bibliográfico de las principales revistas donde se publican artículos del ámbito de la bioantropología en Patagonia Austral. Se analizaron 18 variables en 88 artículos. Los resultados mostraron que, si bien el dato final de la investigación es presentado de manera correcta, no lo es la información que contextualiza ese dato, los metadatos. Se plantea la necesidad de que la comunidad bioantropológica siente unas bases que permitan estandarizar aquello que se quiera compartir en un futuro, con especial atención a los metadatos.
\end{abstract}

Palabras clave: Patagonia Austral; data sharing; bioantropología; restos humanos.

\begin{abstract}
The technical advancements that took place during the last decades have influenced directly the sciences, allowing for the utilization of new research tools that have consequently generated new knowledge. These new developments have bettered the communications and access to information across the globe. In this new framework, data sharing arises as a global trend for the free access to data produced during research investigations. This trend is not well developed for Patagonia Austral. However, before sharing data, it is necessary to know what information can and want to be shared among scientists. To gain a better understanding of what information is available for Patagonia Austral that would allow the implantation of data sharing for this region, we reviewed some the major journals in which articles on archaeological human remains from Southern Patagonia are published. Specifically, we consider 88 articles and evaluated 18 variables. The results showed that, although the data presented in the original papers are correct, the contextual information of those data is not presented accordingly. This contextual information is known as metadata. It is necessary that the community of bio-anthropologists lay the groundwork to standardize the information that would be shared in the future, with spatial attention to the metadata.
\end{abstract}

Keywords: Patagonia Austral; data sharing; bioanthropology; human remains. 
Durante las últimas décadas, los avances tecnológicos han contribuido al aumento exponencial en la generación de nuevas investigaciones y datos científicos (Uhlir y Schröeder 2007; Kullmer 2008; Borgman 2012; Milia et al. 2012; Torres-Salinas et al. 2012; Reed et al. 2015). La bioantropología no ha quedado al margen, haciendo uso de nuevos enfoques metodológicos como los estudios de ADN, isótopos estables (IE) o herramientas computacionales (Kullmer 2008); todos ellos son elementos que han ayudado a profundizar e incrementar la información que se puede obtener de una misma muestra. Este es un asunto transcendental para los investigadores que trabajan con restos arqueológicos o fósiles, donde la disponibilidad de muestra es limitada. Este hecho, se ha visto acompañado por un aumento de publicaciones en el ámbito de la bioantropología, la aparición de nuevas revistas y la apertura de espacios en los cuales los científicos intercambian y confrontan opiniones, información y argumentos. En la tendencia global de profundizar en la información obtenida a partir de los restos óseos humanos, comienza a tomar protagonismo el intercambio de datos o data sharing. Corriente que propone la libre puesta en disposición de los datos producto de las investigaciones científicas, que podría permitir crear grandes bancos de datos, aumentar la eficiencia y calidad de los trabajos o evitar duplicaciones, hecho que se suele acompañar de una reducción de costos. De acuerdo con Delson et al. (2007), esta tendencia se está convirtiendo en un tema central para la comunidad bioantropológica a nivel mundial. Sin embargo, como señalan González y Beguelín (2013), no está desarrollada en la región del cono sur. Para su discusión y posible implementación en el área de Patagonia Austral ${ }^{1}$ (PA), es necesario, en primer lugar, conocer la situación en que se encuentra la información concerniente a la disciplina. Como un aporte a su discusión en la región, consideramos necesario focalizar en algunos aspectos, haciendo visible particularidades locales, en el ámbito de la bioantropología.

Las particularidades locales e históricas que atraviesan las interacciones humanas en las cuales estamos implicados, plantean desafíos al tratar de interpretar cómo pueden afectar cambios en un nivel de análisis al resto de los niveles. Los cambios e innovaciones pensadas en términos lineales de causa-efecto pueden en algunos casos generar interpretaciones simplistas sobre la complejidad de los sistemas abiertos de los cuales formamos parte. Estos sistemas abiertos requieren aproximaciones que consideren de partida solapamientos, imbricaciones y otras configuraciones asociables a fenómenos no lineales (García 2006; Najmanovich 2007). Desde esta perspectiva, el intercambio de información está incluido dentro de los entramados y dinámicas de vínculos que presentamos los seres humanos.

\footnotetext{
1 Últimas estribaciones del continente americano, la isla grande de Tierra del Fuego y los archipiélagos fueguinos. Comprenden las provincias argentinas de Santa Cruz y Tierra del Fuego, Antártida e Islas del Atlántico Sur y la región chilena de Magallanes y la Antártica Chilena.
}

\section{Data sharing}

El intercambio de datos aboga por la puesta a disposición de la comunidad, de manera libre, equitativa, con los menores costes y restricciones, de los datos que son producto de las investigaciones científicas (Azberger et al. 2004; Uhlir y Schröeder 2007; Torres-Salinas et al. 2012). Esta tendencia, propone hacer públicos los datos finales producto del proceso científico, así como el contexto que rodea a esos datos; lo que ha venido a denominarse metadatos (Nelson 2009; Borgman 2012; Torres-Salinas et al. 2012). No se limita al intercambio de la información, atiende a la disponibilidad y preservación de los datos. Está, por tanto, relacionado directamente con la provisión de acceso para el uso y reutilización de los datos (Tenopir et al., 2011). Actualmente, existe cierto consenso acerca de los beneficios del data sharing. Se lo percibe como una parte significativa del actual proceso de producción científica, un factor importante para el progreso de la investigación; llegándose a señalar como una prioridad y un pilar del sistema de investigación. Desde esta perspectiva, se considera que los datos tienen valor más allá del proyecto y el uso previsto para el cual fueron tomados (Azberger et al. 2004; Uhlir y Schröeder 2007; Pinowar et al. 2008; Nelson 2009; Smith 2009; Contreras 2010; Boulton et al. 2012; Milia et al. 2012; Torres-Salinas et al. 2012; Destro Bisol et al. 2014b, 2014c). Como señalan Faniel y Zimmerman (2011), el objetivo es hacer la información accesible y utilizable a cualquier persona, en cualquier momento, en cualquier lugar, y para cualquier propósito.

El intercambio de datos tiene una implementación y expansión desiguales. En algunos campos como meteorología, geofísica, astronomía, biología molecular, ecología o ciencias computacionales, hay un mayor desarrollo (Azberger et al. 2004; Nelson 2009; Contreras 2010; Pinowar 2010; Destro Bisol et al. 2014a, 2014b); destacando las investigaciones relacionadas con la genética, donde se llega a publicar el $45 \%$ de los datos en código abierto (Pinowar 2011). De esta manera, es posible plantear que su implementación ha sido heterogénea, siendo más la excepción que la regla. En el campo de la antropología, como indican Destro Bisol et al. (2014c), la existencia de instituciones con una larga tradición, de pequeñas dimensiones y estructura sencilla, han permitido implementar modelos de libre acceso en todas sus actividades (investigación, reuniones de trabajo y publicaciones) con mayor celeridad que instituciones de mayor tamaño que caracterizan otras disciplinas. Sin embargo, también en la bioantropología, es necesario generar plataformas de trabajo y experiencias exploratorias, que ayuden a promover espacios de diálogo entre los actores interesados, quedando un largo camino por recorrer (Elton y Cardini 2008; Kullmer 2008; González y Beguelín 2013).

Entre los beneficios que se le atribuyen al data sharing: 1) permite a otros autores verificar resultados e hipótesis mediante la replicación de los experimentos, resultando 
en una mayor transparencia y minimizando el riesgo de duplicaciones con los consiguientes beneficios temporales y económicos (Azberger et al. 2004; Delson et al. 2007; Uhlir y Schröeder 2007; Pinowar 2011; Tenopir et al. 2011; Torres-Salinas et al. 2012). El hecho de que los resultados estén a disposición del público los hace más fiables, debido a que son recogidos, integrados y procesados sistemáticamente teniendo en cuenta los estándares de amplias comunidades científicas; situación que desalienta el desarrollo de prácticas no rigurosas (Smith 2009; Borgman 2012; Destro Bisol et al. 2014b). 2) Posibilita el ensamblaje de enormes conjuntos de datos provenientes de distintas fuentes, ofreciendo un aumento de los rangos de análisis, integración y precisión; pero fundamentalmente, permitiendo explorar relaciones y patrones desconocidos, conduciendo hacia nuevas percepciones, hipótesis y descubrimientos, aumentando así el potencial de los datos (Azberger et al. 2004; Delson et al. 2007; Smith 2009; Pinowar 2010; Borgman 2012; Boulton 2012; Destro Bisol et al. 2013, 2014b). A la vez, fomenta la interdisciplinariedad (Uhlir y Schröeder 2007). 3) Al extender el valor de los datos hacia nuevos horizontes, aumenta la eficiencia y calidad de estos acompañándose, normalmente de una reducción de costos (Uhlir y Schröeder 2007; Pinowar 2011; Tenopir et al. 2011). 4) Las publicaciones que tienen en cuenta las prácticas del data sharing obtienen un mayor índice de citación (Eysenbach 2006; Piwowar 2011). El impacto de las citaciones puede ser considerado en futuras decisiones, debido a que este es uno de los parámetros que aumentan la reputación de las instituciones académicas (Pinowar et al. 2008). 5) El paso del tiempo y un uso frecuente de los materiales de estudio puede provocar daños en estos, aun de manera inintencionada. Aparte de reducir el tiempo y los recursos, se evita la degradación de las muestras (Elton y Cardini 2008; Destro Bisol et al. 2014b). 6) La liberación facilita el acceso a los datos provenientes de muestras de las cuales determinadas regiones carecen, como pueden ser muestras biológicas o fósiles (Elton y Cardini 2008; Boulton et al. 2012). Por otro lado, ofrece la oportunidad a países y equipos con menores recursos económicos y tecnológicos acceder a datos obtenidos con los últimos avances metodológicos, a los cuales normalmente no tienen acceso (Uhlir y Schröeder 2007; Boulton et al. 2012; González y Beguelín 2013). 7) Por último, podría ayudar que toda una comunidad de nuevos investigadores se involucre y que las nuevas generaciones puedan educarse, entrenar y practicar a partir de los bancos de datos (Elton y Cardini 2008; Pinowar 2011; Tenopir et al. 2011).

Sin embargo, no todo son beneficios. Existen barreras de tipo ético, personal y técnico. 1) En primer lugar, hay límites por motivos de seguridad nacional, secretos comerciales o privacidad; siendo este último el que más atención ha recibido, fundamentalmente cuando los datos provienen de individuos vivos. Entran en juego los derechos a la privacidad y confidencialidad que rige en la biomedicina bajo juramento hipocrático (Nelson 2009; Tenopir et al. 2011; Destro Bisol et al. 2014a, 2014b). 2) Algunos factores sociales, organizativos y legislativos plantean tensiones o barreras (Hendriks 1999; Azberger et al. 2004; Pinowar 2010). Los científicos a menudo tienen fuertes alicientes para mantener la confidencialidad, al menos hasta el momento de la publicación; incentivos apoyados e incluso ordenados por los procedimientos y políticas de las instituciones donde trabajan (Contreras 2010). 3) LoS espacios de diálogo entre investigadores para obtener datos comparables (estandarizados) requieren esfuerzos extras de revisión, autorización, subida y publicación, acciones que implican tiempo y pericia (Azberger et al. 2004; Pinowar 2010; Frank et al. 2015; Destro Bisol et al. 2014a; MacMillan 2014). Estos aspectos, pueden presentar mayor o menor relevancia en grupos pequeños o en campos con escasa financiación (Destro Bisol et al. 2014b). Este esfuerzo se ve aún más limitado por la falta de reconocimiento de la comunidad científica hacia esta labor (Pinowar 2010; Chavan y Penev 2011; Kansa y Kansa 2013; MacMillan 2014). Esto conlleva, que con frecuencia los metadatos estén pobremente documentados o sean prácticamente inexistentes, a pesar de su importancia para un buen desarrollo del data sharing (Chavan y Penev 2011; MacMillan 2014). 4) A nivel individual es posible considerar una variedad de argumentos y temores que pueden estar presentes o en el imaginario de los autores: a) la posibilidad de que el trabajo desarrollado sea refutado al ser reanalizado, debido a errores en el estudio original, una incorrecta interpretación, o simplemente por una redefinición de la metodología. b) Al hacerse público, otros autores pueden descubrir relaciones adicionales y limitar futuras oportunidades de publicación. c) Asociado a los dos anteriores, algunos autores, consideran que pierden el control sobre sus datos (Pinowar 2010; Destro Bisol et al. 2014a; MacMillan 2014). 5) Por último, las limitaciones tecnológicas persisten, a pesar de los avances señalados al inicio. Concretamente, el acceso a los datos requiere de infraestructuras apropiadas, amplio consenso en la interoperabilidad y efectivos controles de calidad (Azberger et al. 2004); sin embargo, las infraestructuras y repositorios son escasos (Torres-Salinas 2010; Kansa y Kansa 2013). Al mismo tiempo, los cambios tecnológicos plantean la necesidad de continuas adaptaciones a los nuevos soportes y programas, formatos de almacenamiento de datos, hardware de los datos de acogida y software (MacMillan 2014).

Por otro lado, existen una serie de características de índole metodológico-técnico que tienen que ver con la implementación del data sharing que son propias de cada disciplina y del contexto en que éstas se desarrollan. Se pueden agrupar en qué y cómo compartir los datos. El qué, hace referencia al tipo de información que se ha de compartir y el cómo, a la forma de hacerlo. Antes de llegar al cómo, se ha de tener claro el qué. Los datos que se quieran compartir no sólo han de ser accesibles sino inteligibles y de calidad, para permitir su reutilización. Es necesario que se recojan, integren y procesen, teniendo en cuenta los estándares de cada una de las disciplinas; 
buscando un formato estandarizado, con una semántica y estructura específicas. Esta forma de proceder ayudará a la construcción de conjuntos de datos más adecuados para la realización de nuevos análisis y facilitará la integración de datos de diversa procedencia (Nelson 2009; Tenopir et al. 2011; Borgman 2012; Boulton 2012; Kansa y Kansa, 2013; Reed et al. 2015). En relación con la estandarización, resulta importante recuperar la idea de proceso que se incorpora a la discusión como etapas o acuerdos operativos, que va cambiando a lo largo del tiempo, dependiendo de la disciplina. Sin embargo, mientras esto sucede, se sigue generando conocimiento. Por tanto, ese qué irá cambiando y transformándose con el avance científico; en ocasiones, a la par y/o tras los procesos de estandarización; otras veces, fundamentalmente aquellas en los que los procesos de estandarización son complejos, o no se producen, por delante.

Por último, independientemente de los ritmos de implementación del data sharing en las distintas disciplinas, hay un factor común a todas ellas, la importancia de la información contextual, los metadatos. La información contextual es aquella que da origen y rodea al dato, debiendo estar documentada y especificada. Esta información y su disponibilidad es clave para todo aquel que pretenda hacer uso de ella (Schoefield et al. 2009; Nelson 2009; Borgman 2012). Su desarrollo es importante para interpretar las implicaciones de los conjuntos de datos, los procesos de investigación que los produjeron, así como el dato en sí mismo (Hendriks 1999; Borgman et al. 2012; Boulton et al. 2012). En este sentido, como indica Borgman (2012), los resultados científicos a menudo dependen más de la interpretación que de los datos en sí. La explicitación de los metadatos contribuye a ajustar esas interpretaciones al contar con más información (Torres-Salinas et al. 2012).

\section{Implementación del data sharing en la bioantropología de $P A$}

Las trayectorias de las comunidades bioantropológicas plantean diferentes desafíos al momento de tratar con esta problemática en cada lugar. En el ámbito de la bioantropología la existencia de estándares y consensos de cuál ha de ser la forma de abordar esta temática es escasa o nula (Kullmer 2008; Summer y Riddle 2009). Otros campos de conocimiento próximos, como la arqueología, a pesar de no presentar estándares debido a la amplia variedad de metodologías y perspectivas existentes, si muestra una serie de recomendaciones y guías (Frank et al. 2015; Reed et al. 2015). Es en estas situaciones de escaso o inexistente consenso, donde el papel de los metadatos adquiere una mayor relevancia (Frank et al. 2015).

En lo concerniente a la región, se vienen realizando diferentes tentativas con la intención de generar espacios que permitan consensuar de forma periódica procedimientos y criterios para el relevamiento de datos. Se trata de esfuerzos necesarios para el desarrollo de la disciplina, siendo estos los escenarios adecuados para comenzar a discutir acerca del data sharing. Hasta el momento, el desarrollo de esta corriente no ha sido abordado en la región (González y Beguelín 2013). Por tanto, el conocimiento que se tiene acerca de cuál es la situación de ese qué es exiguo. Una primera aproximación a la problemática sería categorizar la información teniendo en cuenta los tipos de estudios que tradicionalmente se desarrollan dentro del ámbito de la antropología biológica: determinación del perfil biológico (sexo, edad, estatura, tafonomía, preservación), paleopatología, paleohistología, morfometría clásica o geométrica, IE, genética, datación. De manera intrínseca a estos, se encuentran los metadatos, en dos niveles diferenciados; un primer nivel, asociado a la muestra en estudio (tipo de muestra, contexto, ubicación); y un segundo nivel, inherente a análisis específicos (datación, IE, genética). En este caso, los metadatos serían la metodología, que muestra se analizó, que laboratorio, etc.

En algunos de los distintos tipos de estudios indicados existen estandarizaciones, o al menos aproximaciones, sobre cuáles son los datos y análisis que han de ser tenidos en cuenta. También, existen bases de datos de distintos campos disciplinares, que, en mayor o menor medida, están vinculados a la bioantropología. Para el análisis del perfil biológico y de la morfometría se encuentra Standars for data collection from human skeletal remains (Buikstra y Ubelaker, 1994); en paleopatología se encuentran bases internacionales como Wellcome Osteological Research Database (http://archive.museumoflondon. org.uk/Centre-for-Human-Bioarchaeology/Database/); en genética GenBank (http://www.ncbi.nlm.nih.gov/ genbank/); o en el campo de la antropometría, Human Bone Morphology Database (https://www.qut.edu.au/ research/research-projects/human-bone-morphologydatabase). Estas bases de datos, al ser de escala global y focalizar su atención en los campos a los que pertenecen, es decir, genética, morfometría, paleopatología, etc. no suelen prestar la atención debida a los metadatos. Como señalan Tenopir et al. (2011), existe cierto grado de satisfacción con las herramientas que permiten la recolección y análisis de datos, pero no así con los soportes de creación y preservación de metadatos, dificultando su intercambio.

\section{Objetivo}

A este punto, se pretende realizar un primer diagnóstico de la situación en la que se encuentra ese qué compartir, en un conjunto de líneas de evidencias vinculadas al estudio de las poblaciones humanas del pasado en PA; prestando especial atención a los metadatos. A tal fin, se llevó a cabo un análisis bibliográfico de cinco publicaciones periódicas que suelen ser las elegidas por quienes redactan trabajos donde se analizan los restos óseos humanos de la región.

\section{Material y métodos}

Para la elaboración de este análisis exploratorio, se eligieron 
cinco publicaciones de ámbito regional e internacional en las que se ha abordado la bioantropología de PA. Dos de lengua inglesa: American Journal of Physical Anthropology (AJPA) creada en 1918 e International Journal of Osteoarchaeology (IJOS) editada por primera vez en 1991; y tres en castellano: Intersecciones en Antropología (IA) cuyo primer número vio la luz en 2000; Revista Argentina de Antropología Biológica (RAAB) fundada en 1996 y la actual Magallania (M) y antigua Serie Ciencias Humanas de Anales del Instituto de la Patagonia, cuyo primer número data de 1970, si bien el primer trabajo del área data de 1973. No se analizaron las actas de congresos y simposios, debido a que en ocasiones presentan una compilación de resúmenes, los cuales no permiten un análisis profundo por su brevedad y carácter sintético. De la misma manera, no se tuvieron en cuenta tesis doctorales y libros, por el carácter exploratorio de este trabajo.

Fueron seleccionados todos aquellos artículos que, o bien señalan la existencia de restos humanos o desarrollan algún tipo de estudio en relación con estos. No se tuvieron en cuenta, las publicaciones cuyos datos fueron extraídos de poblaciones actuales o de individuos vivos. Se analizaron todos los artículos publicados hasta el primer semestre de 2014. Suman un total de 88: 58 de M, 10 de IA, 8 de RAAB, 8 de AJPA y 4 de IJOS (Información suplementaria, Anexo 1).

El estudio contempla el análisis de 18 variables, agrupadas en 7 conjuntos (Información suplementaria, Anexo 2):

1. Revistas publicadas: las ya indicadas

2. Año de publicación: categoriza las publicaciones en cuatro periodos que abarcan periodos de 10 años cada uno, desde la primera publicación de 1973 hasta la actualidad.

3. Tipos de estudios: arqueológicos, genéticos, IE, paleopatológicos, morfométricos o bioantropológicos, en éste se incluyen los análisis del perfil biológico (determinación del sexo, la edad, la estatura o el análisis tafonómico) combinados o no con algunos de los estudios anteriores. Por tanto, es una categoría donde se encuadran trabajos de índole inter y multidisciplinar.

4. Información contextual de la muestra: siete variables:

4.a. Tipo de muestra: puede ser acotada (proveniente de un único yacimiento), o un conglomerado (analiza muestras de varios yacimientos). Dentro de los conglomerados, hay tres tipos: regionales (se restringen a PA); continentales (usan muestras de otras partes del continente); o supracontinentales (incluyen otros continentes).

4.b. Procedencia de la muestra: encontramos tres variables:

- Geográfica: artículos que indican un origen amplio o una región como por ejemplo: Norte de
Tierra del Fuego o Canal Beagle; como aquellos que señalan el yacimiento.

- Georreferenciada: se apunta la georreferenciación exacta del lugar, mediante la latitud y longitud.

- Étnica: se desconoce la procedencia, pero si la etnia a la que pertenecieron los restos (Selk'namHaus, Yámana, Kawésqar y Aonikenk), utilizando ésta como un indicador geográfico, debido a que cada una de ellas se asocia a un área.

Las tres variables pueden adquirir los mismos estados, se conoce la procedencia de todos los individuos (en el caso de la variable geográfica, se diferencia entre los que indican el yacimiento o la región), sólo el de algunos o el de ninguno. Existen casos, en los que son los autores los que señalan que esta información se desconoce en uno o varios individuos de la muestra. La otra variante posible, es que se indique la procedencia de los sujetos analizados en ese trabajo, pero no así las muestras con las que se comparan y discuten de trabajos anteriores.

4.c. Disposición actual de los restos:

- Localización: institución o emplazamiento en el que se albergan en el momento del estudio los restos. Pueden indicarse todos los individuos, algunos o ninguno. En alguna ocasión se señala en los agradecimientos.

- Individuo: explicitación de los restos óseos analizados, bien por el nombre que se le dio al ser excavado o por el número que se le da al ser incluido en las colecciones de instituciones. Se tiene en cuenta si se señalan todos, algunos o ninguno.

5. Datación: agrupa 4 variables: la muestra utilizada para hacer el estudio; el laboratorio que lo realiza; la técnica empleada; y el código de identificación que le da el laboratorio que realiza el análisis a la muestra. Los estados son comunes a todas ellas: se señala en todos los casos (en el caso del laboratorio se puntualiza si son uno o varios, los laboratorios que realizan el análisis), en algunos casos, en ninguno o sólo los del trabajo en cuestión. Por otro lado, están los casos en los que se indica la datación, pero esta ha sido realizada en un trabajo anterior, pudiendo señalarse alguna de las cuatro variables en cuestión o no.

6. IE: aglomera 3 variables, las mismas que en el caso de la datación con la excepción de la técnica. Los estados también se repiten, menos el del rango.

7. Genética: compuesto por 2 variables: muestra y código de identificación. Los estados que pueden adquirir estas variables son los mismos que corresponden a la datación y a los IE.

Cabe señalar que no en todas las publicaciones se analizaron las 18 variables, debido a que algunas de ellas son específicas de determinados tipos de estudios, como 
los casos de IE o genética.

Con el objeto de realizar una valoración descriptiva de las variables cualitativas y las relaciones que puedan existir entre estas, se llevó a cabo un análisis de frecuencias de cada una de ellas de manera individualizada y los cruces existentes. Para desarrollar estos análisis y elaborar los diagramas correspondientes se hizo uso del programa $\mathrm{R}$ (R Development Core Team 2008).

\section{Resultados}

El número de publicaciones que tienen en cuenta los restos óseos humanos en PA ha aumentado a través del tiempo (Tabla 1), destacando el último periodo (2004-2014) en el cual se concentran el 48,9\% de las publicaciones. El aumento de publicaciones en los dos últimos periodos coincide con la aparición de las revistas RAAB, IJOS e IA. Si bien fue y continúa siendo Magallania la que más espacio dedica a las publicaciones referidas a los restos óseos humanos de la región.

De la misma manera que se observa un aumento de trabajos a través del tiempo, se aprecia una mayor

Tabla 1: Relación entre las variables Tiempo y Revista

Table 1: Relationship between the variables time and journal

\begin{tabular}{lcccrr}
\hline & \multicolumn{5}{c}{ Año de publicación } \\
\cline { 2 - 5 } & $1973-1983$ & $1984-1993$ & $1994-2003$ & $2004-2014$ & \multirow{2}{*}{$\Sigma$} \\
\cline { 2 - 5 } & $\mathrm{n}(\%)$ & $\mathrm{n}(\%)$ & $\mathrm{n}(\%)$ & $\mathrm{n}(\%)$ & \multicolumn{1}{c}{$5(5,7)$} \\
\hline RAAB & - & - & $3(3,4)$ & $5(9,1)$ \\
M & $4(4,6)$ & $15(17)$ & $19(21,6)$ & $20(22,7)$ & $58(65,9)$ \\
IJOS & - & - & & $4(4,6)$ & $4(4,6)$ \\
IA & - & - & $1(1,1)$ & $9(10,2)$ & $10(11,3)$ \\
AJPA & - & - & $3(3,4)$ & $5(5,7)$ & $8(9,1)$ \\
\hline \multicolumn{1}{r}{$\Sigma$} & $4(4,6)$ & $15(17)$ & $26(29,5)$ & $43(48,9)$ & $88(100)$ \\
\hline
\end{tabular}

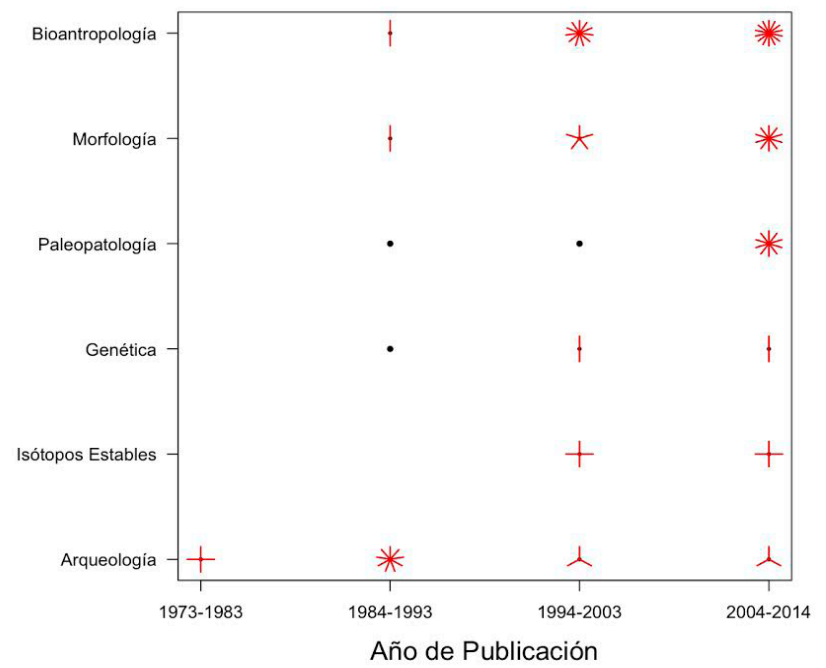

Figura 1: Sunflower plot donde se muestra la relación entre año de publicación y tipo de estudio. En este gráfico, el número de radios representan la cifra de trabajos publicados

Figure 1: Sunflower plot showing the relationship between year of publication and study type. In this graph, the radii number represents the quantity of published manuscripts diversidad en los tipos de análisis que se desarrollan sobre los huesos (Fig. 1). Esta tendencia se caracteriza por una reducción de los trabajos arqueológicos, no sólo en número sino con respecto al conjunto de trabajos analizados.

Por otro lado, se observan diferencias entre los tipos de análisis que se publican según la revista. Aquellas editadas en castellano, y más cercanas a la zona de estudio, abarcan un mayor espectro de tipos de estudios, siendo Magallania, la única que recoge todo tipo de estudios. Las revistas en lengua inglesa se centran en estudios específicos, morfometría y genética en AJPA e IE y bioantropológicos, en IJOS (Fig. 2).

En lo que concierne a los metadatos de primer nivel, y específicamente al tipo de muestra (Tabla 2), se observa que cerca de la mitad de los trabajos desarrollados $(47,7 \%)$ se ha realizado sobre muestras provenientes de un yacimiento acotado. Del resto, que utiliza muestras de distintos orígenes, se destacan los análisis de restos esqueletales de la región, con el 32,9\% del total. Sin embargo, el uso del tipo de muestra depende de la disciplina, los análisis de genética o morfología analizan siempre conglomerados, destacando el uso de conglomerados supracontinentales en morfología, siendo el 58,8\% (10 de 17) de los trabajos analizados. El resto de las categorías muestran una situación intermedia, destacando bien el conglomerado regional o el acotado, teniendo más peso la muestra acotada en los trabajos de

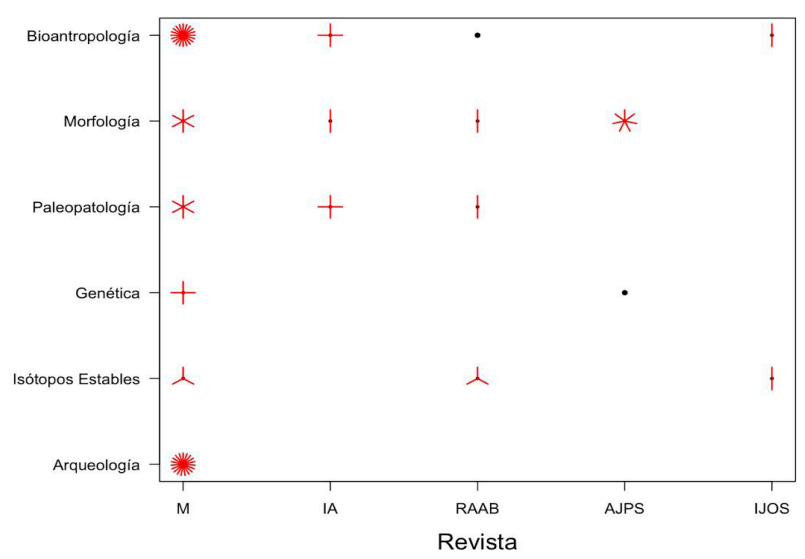

Figura 2: Sunflower plot donde se muestra la relación entre revista y tipo de estudio. En este grafico el número de radios representan la cifra de trabajos publicados. Revistas: M: Magallania; IA: Intersecciones en Antropología; RAAB: Revista Argentina de Antropología Biológica; AJPA: American Journal of Physical Anthropology; IJOS: International Journal of Osteoarchaeology

Figure 2: Sunflower plot showing the relationship between journal and study type. In this graph, the radii number represents the quantity of published manuscripts. Journals: M: Magallania; IA: Intersecciones en Antropología; RAAB: Revista Argentina de Antropología Biológica; AJPA: American Journal of Physical Anthropology; IJOS: International Journal of Osteoarchaeology 


\begin{tabular}{|c|c|c|c|c|c|c|}
\hline & & \multicolumn{5}{|c|}{ Tipo de muestra } \\
\hline & & Acotada & Con. regional & Con. continental & Con. supracontinental & \multirow{2}{*}{$\Sigma$} \\
\hline & & $n(\%)$ & $n(\%)$ & $n(\%)$ & $n(\%)$ & \\
\hline \multirow{6}{*}{ 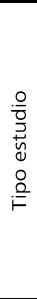 } & Arqueología & $\begin{array}{c}14 \\
(15,9)\end{array}$ & $5(5,7)$ & - & - & $\begin{array}{r}19 \\
(21,6)\end{array}$ \\
\hline & Isótopos estables & $2(2,3)$ & $6(6,8)$ & - & - & $8(9,1)$ \\
\hline & Genética & - & $4(4,6)$ & - & $1(1,1)$ & $5(5,7)$ \\
\hline & Paleopatología & $5(5,7)$ & $7(7,9)$ & - & - & $\begin{array}{r}12 \\
(13,6)\end{array}$ \\
\hline & Morfología & - & $1(1,1)$ & $6(6,8)$ & $10(11,4)$ & $\begin{array}{r}17 \\
(19,3)\end{array}$ \\
\hline & Bioantropología & $\begin{array}{c}21 \\
(23,9)\end{array}$ & $6(6,8)$ & - & - & $\begin{array}{r}27 \\
(30,7) \\
\end{array}$ \\
\hline \multirow{6}{*}{$\begin{array}{l}0 \\
\mathscr{o} \\
\dot{0} \\
\vdots \\
\vdots\end{array}$} & No se indica & & & - & $1(1,1)$ & $1(1,1)$ \\
\hline & Si, yacimiento & $\begin{array}{c}39 \\
(44,3)\end{array}$ & $22(25)$ & $1(1,1)$ & - & $\begin{array}{r}62 \\
(70,4)\end{array}$ \\
\hline & Si, región/área & - & $6(6,8)$ & $5(5,7)$ & $10(11,4)$ & $\begin{array}{r}21 \\
(23,9)\end{array}$ \\
\hline & Se indican algunos & - & $1(1,1)$ & - & - & $1(1,1)$ \\
\hline & Desconocida & $1(1,1)$ & - & - & - & $1(1,1)$ \\
\hline & Si, pero hay desconocidos & $2(2,3)$ & - & - & - & $2(2,3)$ \\
\hline \multirow{6}{*}{ 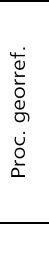 } & No se indica & $\begin{array}{c}24 \\
(27,3)\end{array}$ & $24(27,3)$ & $6(6,8)$ & $11(12,5)$ & $\begin{array}{r}65 \\
(73,9)\end{array}$ \\
\hline & Se indica & $\begin{array}{c}17 \\
(19,3)\end{array}$ & $1(1,1)$ & - & - & $\begin{array}{r}18 \\
(20,4)\end{array}$ \\
\hline & Unicamente los de este trabajo & - & $1(1,1)$ & - & - & $1(1,1)$ \\
\hline & Si, pero no los de estudios anteriores & - & $3(3,4)$ & - & - & $3(3,4)$ \\
\hline & Desconocida & $1(1,1)$ & - & - & - & $1(1,1)$ \\
\hline & $\Sigma$ & $\begin{array}{c}42 \\
(47,7)\end{array}$ & $29(32,9)$ & $6(6,8)$ & $11(12,5)$ & $\begin{array}{r}88 \\
(100) \\
\end{array}$ \\
\hline
\end{tabular}

Tabla 2: Relación entre las variables tipo de muestra y tipo de estudio, y procedencia geográfica y procedencia georreferenciada

Table 2: Relationship between sample type and study type, and geographic origin and georeferenced origin

acentúa, existiendo un $75 \%$ de los trabajos que no lo indica (Tabla 2). Encontrándose el 25\% restante, fundamentalmente, en dos tipos de estudios: arqueológicos y bioantropológicos. La georreferenciación está limitada prácticamente a ciertos trabajos con muestra acotada (16 de los 42) y son sólo 5 de 46, los casos de conglomerados que lo señalan (Tabla 3). El otro elemento que ha sido utilizado como indicador de procedencia, más allá de la geografía, ha sido la etnia; si

arqueología y bioantropología.

Se aprecia un descenso de la información acerca de la procedencia, según aumenta el área que ocupa el conglomerado. La relación del tipo de muestra con respecto a la procedencia geográfica evidencia cómo en los trabajos que analizan muestras acotadas y conglomerados regionales se da información acerca del yacimiento; mientras que en los conglomerados continentales y supracontinentales se usa información más genérica acerca de la región de la que provienen los restos analizados. Cuando la comparación se realiza con la variable procedencia georreferenciada, esta tendencia se bien, este uso se ha visto drásticamente reducido durante la última década. (Tabla 4).

En lo que respecta a la disposición actual de los restos óseos humanos utilizados, se observa que el 48,9\% no señalan localización y que el $36,3 \%$ no indican qué individuos analizan. Un 26,2\% tiene en cuenta ambos parámetros, aunque sea en los agradecimientos, un $19,3 \%$ no tiene en cuenta ninguno de los dos; el restante $54 \%$ omite algún tipo de información (Tabla 5).

En el caso de la localización no se aprecia ninguna tendencia a través del tiempo. En la variable individuo, el
Tabla 3: Relación entre las variables tipo de estudio y procedencia georreferenciada

Table 3: Relationship between study type and georeferenced origin

\begin{tabular}{lccccccc}
\hline & \multicolumn{7}{c}{ Tipo estudio vs Procedencia georreferenciada } \\
\cline { 2 - 8 } & Arqueo. & IE & Genética & Paleopat. & Morfo. & Bioantrop. & \multirow{2}{*}{$\Sigma$} \\
\cline { 2 - 8 } & $\mathrm{n}(\%)$ & $\mathrm{n}(\%)$ & $\mathrm{n}(\%)$ & $\mathrm{n}(\%)$ & $\mathrm{n}(\%)$ & $\mathrm{n}(\%)$ & \\
\hline No se indica & $10(11,4)$ & $7(7,9)$ & $4(4,6)$ & $11(12,5)$ & $17(19,3)$ & $16(18,2)$ & $65(73,9)$ \\
Se indica & $7(7,9)$ & $1(1,1)$ & - & $1(1,1)$ & - & $9(10,2)$ & $18(20,4)$ \\
Se-indican-algunos & - & - & $1(1,1)$ & - & - & - & $1(1,1)$ \\
Únicamente-los-de-este-trabajo & $2(2,3)$ & - & - & - & - & $1(1,1)$ & $3(3,4)$ \\
Desconocida & - & - & - & - & - & $1(1,1)$ & $1(1,1)$ \\
\hline \multicolumn{1}{c}{$\Sigma$} & $19(21,6)$ & $8(9,1)$ & $5(5,7)$ & $12(13,6)$ & $17(19,3)$ & $27(30,6)$ & $88(100)$ \\
\hline
\end{tabular}

Tabla 4: Relación entre las variables año de publicación y procedencia etnográfica

Table 4: Relationship between year of publication and ethnographic origin

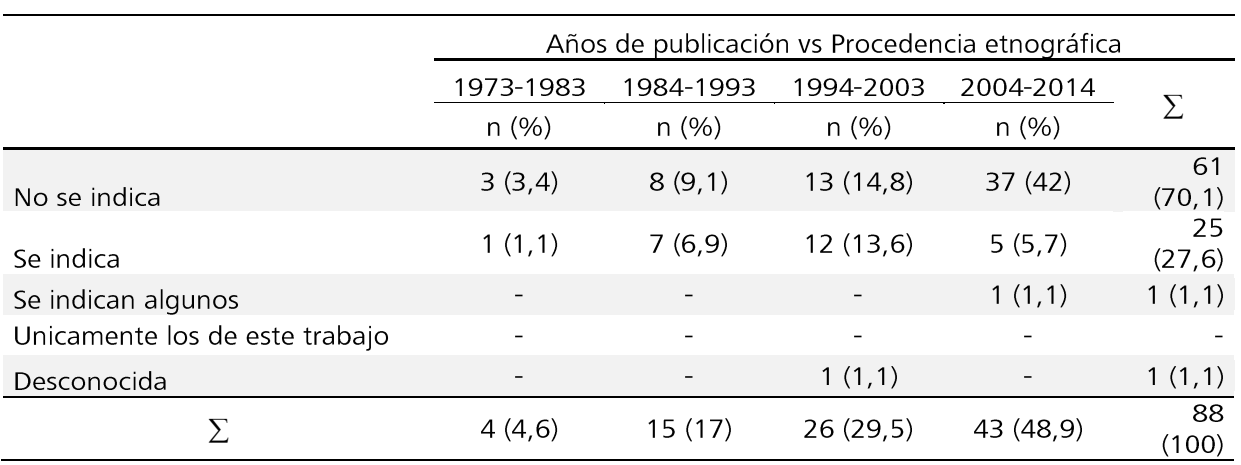




\begin{tabular}{|c|c|c|c|c|c|c|}
\hline & & \multicolumn{5}{|c|}{ Localización } \\
\hline & & No lo indica & Lo indica & Algunos & Agradecimientos & \multirow{2}{*}{$\sum$} \\
\hline & & n (\%) & $\mathrm{n}(\%)$ & n (\%) & n (\%) & \\
\hline \multirow{4}{*}{$\begin{array}{l}\stackrel{\geq}{\overline{0}} \\
\underline{\Xi}\end{array}$} & No lo indica & $17(19,3)$ & $14(15,9)$ & - & $1(1,1)$ & $\begin{array}{c}32 \\
(36,3)\end{array}$ \\
\hline & Lo indica & $25(28,4)$ & $20(22,8)$ & $3(3,4)$ & $3(3,4)$ & $\begin{array}{c}51 \\
(57,9)\end{array}$ \\
\hline & Algunos & $1(1,1)$ & $4(4,6)$ & - & - & $5(5,7)$ \\
\hline & $\sum$ & $43(48,9)$ & $38(43,2)$ & $3(3,4)$ & $4(4,6)$ & $\begin{array}{r}88 \\
(100)\end{array}$ \\
\hline
\end{tabular}

Tabla 5: Relación entre localización e individuo

Table 5: Relationship between localization and individual

segundo periodo se muestra como una excepción, siendo mayor el número de trabajos que no lo indican que el que lo hacen. Si con relación al tiempo las diferencias no eran notables, sí lo son con respecto al tipo de muestra a analizar. En el caso de la localización, se aprecia cómo en los conglomerados suele estar señalada y en el caso de las muestras acotadas donde no se indica. Por el contrario, en el caso de los conglomerados continentales y supracontinentales no se suele señalar qué muestra se analiza, no así con las muestras acotadas y los conglomerados regionales (Tabla 6).

Los metadatos de segundo nivel muestran distintas particularidades según el tipo de análisis al que nos refiramos (Tabla 7); si bien tienen en común la reutilización de resultados de trabajos anteriores, de los que no suelen señalarse los metadatos. En lo que respecta a los análisis de datación, se puede apreciar: cómo la reutilización de la información se encuentra en torno al 50\% y, por otro lado, cómo la práctica habitual es indicar el resultado final, obviando la información acerca de la muestra en el $53,1 \%$; la técnica en $78,7 \%$; el laboratorio en el 80,8\%; y el identificador en el 53,2\% de los casos. En cuanto a la presentación de análisis de datación propios del trabajo en cuestión, se suele indicar la muestra, pero no así el identificador que le da el laboratorio. Los análisis de IE muestran una reutilización de los datos de trabajos anteriores sin indicar los metadatos en torno al 20-25\%. Es común en este tipo de análisis que se utilicen distintos laboratorios para analizar las muestras y se suele indicar el identificador (65\%). En el caso de los análisis genéticos, cuando el trabajo se centra específicamente en el tema, en la mayoría de los casos (4 de 5) se indica la muestra utilizada y el identificador dado para el análisis específico. Cuando los datos genéticos son incorporados en un trabajo interdisciplinario (aquí denominados bioantropológicos), los metadatos de segundo nivel no son indicados. Estos datos pueden ser interpretados como una tendencia general, sin dejar de tener en cuenta que la muestra considerada para este tipo de estudios es muy baja $(n=7)$.

\section{Discusión}

Las categorías consideradas en este análisis sobre las publicaciones de restos óseos humanos de PA pretendieron caracterizar la información y presentarla como un disparador para futuras discusiones. Los resultados pretenden también instalar la necesidad de estos diagnósticos periódicos, que permitan evaluar tendencias y cambios en el tiempo. Asimismo, es de esperar que las categorías que se utilizaron en este trabajo para agrupar la información puedan ser mejoradas ofreciendo diagnósticos más ajustados. En ningún momento se tiene intención de estandarizar la información, para esto es necesario que sea discutido en el seno de la comunidad científica.

Los resultados obtenidos en este diagnóstico muestran un aumento significativo del número de trabajos realizados en el área, pasando de 4 en la primera década a 43 en la última. Incremento que está en consonancia con el aumento exponencial que se ha producido tanto a nivel general en el ámbito científico (Borgman 2012;

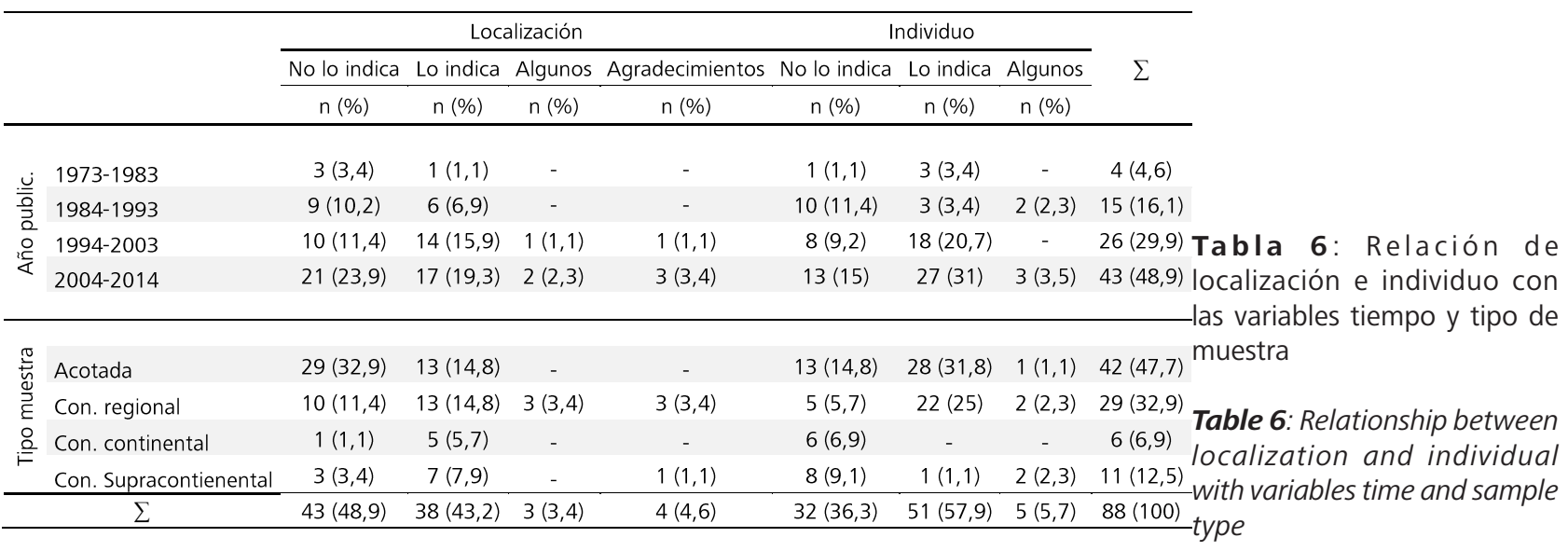


Tabla 7: Número de estudios y frecuencias de los metadatos de segundo nivel. Los guiones señalan los estados no contemplados. Los asteriscos hacen referencia a que el $n$ aquí presentado es el sumatorio de los trabajos de cada tipo de estudio (8 en el caso de IE y 5 en el de genética), más aquellos encuadrados dentro de la categoría de bioantropología

Table 7: Number of studies and frequencies of second level metadata. Dashed lines indicate the states were not completed. The asterisks refer to the fact that the $n$ presented here is the sum of the works of each type of study (8 in the case of SI and 5 in the case of genetics), plus those included in the category of bioanthropology

\begin{tabular}{lccccccccc}
\hline & \multicolumn{4}{c}{ Datación $(n=47)$} & \multicolumn{3}{c}{ Isótopos Estables $(n=20)^{*}$} & \multicolumn{2}{c}{ ADN (n=7)* } \\
\cline { 2 - 10 } & Muestra & Técnica & Laboratorio & Identificador & Muestra & Laboratorio & Identificador & Muestra & Identificador \\
\cline { 2 - 10 } & $n(\%)$ & $n(\%)$ & $n(\%)$ & $n(\%)$ & $n(\%)$ & $n(\%)$ & $n(\%)$ & $n(\%)$ & $n(\%)$ \\
\hline No se indica & $9(19,1)$ & $14(29,8)$ & $16(34)$ & $8(17)$ & $5(25)$ & $5(25)$ & $1(5)$ & $1(14,3)$ & $1(14,3)$ \\
Se indica & $11(23,4)$ & $7(14,9)$ & $4(8,4)$ & $13(27,6)$ & $5(25)$ & $2(10)$ & $11(55)$ & $4(57,1)$ & $4(57,1)$ \\
Varios laboratorios & - & - & $1(2,1)$ & - & - & $8(40)$ & - & - & - \\
Se indican algunos & $1(2,1)$ & - & $1(2,1)$ & - & - & - & - & - & - \\
Unicamente los de este trabajo & $1(2,1)$ & $1(2,1)$ & - & $1(2,2)$ & $4(20)$ & - & $1(5)$ & - & - \\
En otro trabajo, pero indicado & $9(19,1)$ & $2(4,2)$ & $3(6,3)$ & $8(17)$ & $2(10)$ & - & $2(10)$ & $1(14,3)$ & - \\
En otro trabajo, pero sin indicar & $16(34)$ & $23(48,9)$ & $22(46,8)$ & $17(36,2)$ & $4(20)$ & $5(25)$ & $5(25)$ & $1(14,3)$ & $2(28,6)$ \\
\hline
\end{tabular}

Milia et al. 2012), como en la antropología biológica (Kullmer 2008). Esta situación se ha visto acompañada e impulsada por el desarrollo de nuevos tipos de estudios y metodologías que han permitido profundizar en el conocimiento (Kullmer 2008; Schoefield et al. 2009; Tenopir et al. 2011; Torres-Salinas et al. 2012) y que se ve reflejado en PA en la aparición de estudios genéticos, de IE o de morfometría geométrica, en los periodos más recientes (Yesner et al. 1991; Lalueza et al. 1993-1994; González-José et al. 2008). Esta incorporación de nuevas técnicas está acompañada de nuevos encuadres teóricos, mayor especialización profesional en bioantropología, surgimiento de asociaciones profesionales y reuniones científicas a nivel regional y un mayor interés por la conservación preventiva de restos humanos (Carnese y Pucciarelli 2007).

La incorporación de nuevas áreas y el aumento de la inter y multidisciplinariedad se han visto reflejados en el aumento en los dos últimos periodos de los trabajos que aquí hemos definido como bioantropológicos. A la vez que esto sucedía, se ha observado una reducción del peso de los trabajos de arqueología. Pasando de representar un $63 \%$ del total, en el periodo anterior a los años 80 (Guichón y Figuerero Torres 1991), a un 7\% (3 trabajos de 43) en la última década. El desarrollo de estrategias interdisciplinarias se ha observado de igual manera en los trabajos arqueológicos, combinando los saberes arqueológicos con otros campos.

En la última década, se produce un aumento de los estudios de morfología y paleopatología. En el caso de la morfología, los análisis se caracterizan por la utilización de conglomerados, que lleva aparejada una falta de información acerca de los individuos analizados y la procedencia de estos. Esta desatención de los metadatos dificultará trabajos venideros. El crecimiento de estudios paleopatológicos en los últimos 10 años da cuenta del esfuerzo por desarrollar estas líneas de investigación e incorporarlas a las discusiones interdisciplinarias, en parte debido al aumento de especialistas en esta área formados en los años 80 y 90, tras la introducción, por la Dra. Jane Buikstra, de la disciplina en la década de los 80 (Mendonça de Souza y Guichón 2012). En lo que respecta a los estudios genéticos y de IE se mantienen en un número relativamente bajo y constante durante los últimos 20 años. Esto resulta llamativo en el caso de los estudios de ADN antiguo, donde los análisis de restos de PA fueron pioneros (Lalueza et al. 1993-1994). Esta tendencia puede deberse a la falta de impulso en estas áreas, o bien reflejar la preferencia por otras revistas no incluidas en este estudio. En el caso de los estudios de IE se observa que la mayoría de los artículos publicados presentan el Identificador del Laboratorio, lo que en teoría facilita el reconocimiento de la muestra específica que fue analizada; sin embargo, el escaso reporte de metadatos de primer nivel, particularmente la identificación del individuo y la posición georreferenciada, obstaculizan esta tarea. Otra dificultad que surge de nuestro análisis es que no se reporta de manera sistemática el tipo de muestra analizada (solo en 7 casos se indica, 5 en forma original y 2 sobre reportes de otros trabajos). La falta de consenso sobre este reporte entorpece la interpretación de los datos, debido que la información isotópica podría variar tratándose de dientes o huesos (Ambrose 1993; Fry 2006).

\section{Metadatos de primer nivel}

La escasez de procedimientos estandarizados para la recolección de datos en la antropología biológica (Kullmer 2008; Summer y Riddle 2009) condiciona el desarrollo de las bases de qué y cómo compartir los datos (Elton y Cardini 2008; González y Beguelín 2013). Esta situación, Ileva a que en ocasiones no se explicite parte de la información, posiblemente por el desconocimiento de su importancia, sufriendo estas pérdidas los metadatos, como señalan 
los resultados de este diagnóstico. A pesar de que, como se señalaba anteriormente, cuanto más amplios y detallados sean estos, más fácil será la comprensión a posteriori de los datos a los que acompañan (Hendriks 1999; Tenopir et al. 2011). Una escasez de metadatos dificultará el intercambio de datos en su desarrollo inicial, entorpeciendo el posterior almacenamiento y puesta en común, lo que hemos venido llamando el cómo.

Dentro de los metadatos, existen algunos que probablemente tienen mayor transcendencia. En concreto, las variables que tienen que ver con la disposición actual de los restos: localización e individuo. El desconocimiento de estas variables es del $48,9 \%$ en el primer caso y del $36,3 \%$ en el segundo; destacando el caso de los conglomerados continentales y supracontinentales, donde solo 3 de los 17 trabajos aportan información acerca de los individuos. Sin embargo, suelen señalar en qué instituciones se encuentran los restos que han utilizado e incluso el número de sujetos analizados, pero no cuáles. Situación que como ya fue señalado conlleva la imposibilidad de verificar los experimentos, la posible duplicación de trabajos, impidiendo a su vez, la realización de estudios extras que permitan extraer una mayor cantidad de información del mismo material; con el consiguiente gasto de tiempo y costos, aparte de someter al material a un mayor desgaste.

En lo que concierne a la procedencia, cuanto más precisa sea la información, en este caso sería la georreferenciación, mejor será la comprensión de los datos que acompaña y aumentará la calidad de la información ofrecida y su potencial (Smith 2009; Borgman 2012; Boulton et al. 2012; Destro Bisol et al. 2014b). Sin embargo, los datos analizados muestran que según aumenta el espacio que ocupa el conglomerado se reduce la precisión de la información de procedencia; señalándose la georreferenciación sólo en el 25,3\% de los trabajos, siendo los conglomerados en su conjunto los que no atienden este asunto.

Mención aparte merece la utilización de la etnia como indicador de procedencia (Lalueza Fox et al. 1993-1994; González-José et al. 2002). Para este uso, se precisaría de una definición ajustada de los distintos grupos humanos que ocuparon el territorio, los espacios que habitaron, los límites entre estos y su variación en el tiempo (Manzi 2001). Debido a las dificultades que entraña dicha cuestión, algunos autores decidieron la utilización de regiones geofísicas (Canal Beagle, Estrecho de Magallanes, Isla Navarino, norte de la isla grande de Tierra del Fuego, etc.) evitando señalar ninguna connotación étnica, hecho que reflejan los resultados de este análisis. De esta manera, también se intenta eludir una vinculación de aquellos restos datados en fechas tempranas a una etnia particular, al igual que sucede con aquellos individuos de los cuales se desconoce datación (Guichón, 2002).
Respecto a los tipos de estudios analizados en este diagnóstico, algunos de ellos no tienen metadatos inherentes al análisis, teniendo únicamente los de primer nivel. Son los casos de la paleopatología, la morfometría, la arqueología o algunos de los estudios que se desarrollan en la categoría de bioantropología.

\section{Metadatos segundo nivel}

Si bien, la datación es una herramienta aceptada por la comunidad científica, no hay consenso de parte de ésta acerca de qué información brindar cuando se presentan las dataciones. La datación radiocarbónica puede realizarse sobre: hueso, madera o suelo (cualquier sustancia orgánica). Cuando se informa el fechado de un resto óseo humano, se debe aclarar si fue realizado sobre una muestra ósea del mismo (antigüedad del individuo), o de manera indirecta, por asociación a algún otro material como, por ejemplo, sedimento o carbón (antigüedad del sitio o contexto donde se halló al individuo). Otra cuestión omitida, en el $80 \%$ de los casos, fue la aclaración de la técnica empleada para el fechado (siendo las más habituales: Contadores proporcionales de gas [GPC] o acelerador acoplado a espectrometría de masa [AMS]) y el laboratorio en el que se realizó.

En relación con la exactitud del dato, es importante su calibración a años calendarios. En los últimos 3000 años, la producción de ${ }^{14} \mathrm{C}$ fue relativamente constante y por lo tanto se puede considerar similar a los años calendarios. Sin embargo, para aquellas muestras cuya antigüedad superan los 3000 años, es necesaria su calibración con la utilización de la dendrocronología (Pessenda 1998). Pocos trabajos cuyas antigüedades superan dicho límite, aclaran si el fechado presentado fue calibrado o no. Hoy por hoy, se cuenta con programas como CALIB o CALPAL que realizan la conversión de la edad radiocarbónica a los años calendario.

En cuanto al análisis de IE, los metadatos de segundo nivel expuestos en este trabajo representan solo parte de un posible conjunto de información anexa, inherente a estos análisis, que podría ser evaluada. No obstante, los aquí evaluados (muestra, laboratorio, identificador de laboratorio) resultan fundamentales a la hora de compartir datos y lograr un buen uso de estos por parte de la comunidad científica. Entre la información anexa no analizada en este trabajo podemos mencionar como metadatos de segundo nivel: el método que se utilizó para el procesado de la muestra, el tratamiento y la calidad del material y su estado de preservación (Ambrose 1990; Coplen 2011). Esto último, debería ser evaluado por la presentación de porcentajes de los elementos $(\% \mathrm{C}, \% \mathrm{~N})$, o el rendimiento de los mismos (por ejemplo: Oxygen yield en el caso de análisis de ${ }^{18} \mathrm{O}$ sobre apatita o esmalte) para establecer la confiabilidad sobre los valores isotópicos.

Otro aspecto que involucra metadatos de segundo nivel está relacionado al reporte de los datos isotópicos y su 
procesado post-análisis, la normalización de los valores "crudos". Es esencial reportar la precisión del análisis y como esta fue estimada (por ejemplo, análisis de muestras por duplicado, uso de materiales de referencia internos o el valor de precisión estimado por el laboratorio basado en su historial). Asociado a este tratamiento de los datos, es importante registrar y reportar si los valores están estandarizados o 'anclados' a una escala internacional (V-PDB, AIR, V-CDT, V-SMOW, etc.), cómo se realizó esta estandarización y normalización (mediante una corrección de offset o una corrección de 'dos puntos' en el laboratorio de servicios) y qué materiales se utilizaron (por ejemplo, materiales de referencia internos o estándares internacionales) (Coplen y Qi 2009; Coplen 2011).

En el caso de los estudios genéticos, los metadatos de segundo nivel que son necesarios a la hora de considerar los resultados son, en primer lugar, la técnica de análisis $y$, en el caso de la secuenciación, es de fundamental importancia que se indique el rango de lectura, para poder establecer claramente el fragmento de ADN del cual pudo obtenerse información acerca de la presencia/ausencia de polimorfismos. Cuando este dato no se consigna, es imposible discernir si la ausencia de una mutación específica se debe a una ausencia real o a la falta de lectura de la región que la contiene. Otros metadatos de segundo nivel relevantes, por su contribución al desarrollo de este tipo de análisis, son: la explicitación del fragmento óseo o la pieza dental utilizada, la técnica de extracción y el porcentaje de éxitos y fracasos. Por lo general, cuando el trabajo se centra en el análisis de ADN, estos metadatos son consignados.

Los párrafos anteriores dan cuenta de las numerosas variables que presentan los análisis con metadatos secundarios. En este trabajo, se realiza una primera aproximación a la problemática que a futuro debería ser profundizada atendiendo a las variables citadas y a aquellas que se consideren relevantes para cada estudio.

A la luz de los resultados, y tal como señalan algunos autores (Elton y Cardini 2008; Schoefield et al. 2009; González y Beguelín 2013), se considera necesario que la comunidad se reúna periódicamente a debatir fórmulas que permitan estandarizar e implementar procedimientos consensuados, haciendo hincapié en los metadatos, los cuales permiten mejorar la calidad y potencial de los datos a compartir y son indispensables para la reproducción de resultados (Borgman et al. 2012; MacMillan 2014). Sin embargo, los metadatos cuando son tenidos en cuenta suelen estar pobremente documentados (Chavan y Penev 2011; MacMillan 2014), situación que también muestran los resultados de este diagnóstico. El debate sobre estas cuestiones también involucra a los organismos editores e instituciones financiadoras. En este sentido, algunas editoriales e instituciones están comenzando a considerar la problemática, recomendando el intercambio de datos, llegando incluso en algunos casos a exigirlo como condición sine qua non, para la financiación o publicación de los trabajos (NIH 2003; OECD 2007; Schoefield et al. 2009; Pinowar 2011; Torres-Salinas et al. 2012; Holdren 2013). En el ámbito argentino también se ha legislado en esta dirección, la Ley 26899 (Creación de Repositorios Digitales Institucionales de Acceso Abierto, Propios o Compartido) sancionada y promulgada por el Honorable Congreso de La Nación Argentina a finales de 2013 es un ejemplo. A lo largo de los nueve artículos que la conforman, aboga por la libre disposición de la información financiada con fondos públicos y la creación de repositorios y plataformas pertinentes a tal fin (Ley 26899: http://repositorios.mincyt.gob.ar/recursos.php). A pesar de la existencia de esta ley, no se tiene conocimiento de su aplicación en el área de la antropología biológica; quizás debido al desconocimiento de su existencia. Sin embargo, y al contrario que sucede en otras regiones del mundo, las editoriales de nuestro ámbito de investigación en la región aún no están teniendo en cuenta esta corriente, como se puede observar en las guías a los autores de éstas.

En estos momentos, es difícil predecir el desarrollo que tendrán las investigaciones en PA. La implementación o no del data sharing forma parte de esta agenda futura. Los posibles cómo implementar cualquier cambio, entre ellos el data sharing, requieren como señalamos al principio de un conjunto de actores y la consideración de las particularidades locales e históricas que nos atraviesan.

\section{Conclusión}

El uso del data sharing, necesita de una especial atención por parte de los científicos en lo que concierne al trato que se ha de dar a los datos, pero fundamentalmente a los metadatos. En estos momentos, y como señala este análisis, la relevancia que adquieren los metadatos en los trabajos de la disciplina en la región es escasa y no está estandarizado. En lo que respecta a los de primer nivel, la calidad de la información utilizada en cuanto a referencias espaciales y temporales es un problema e influye de manera diferente según el tipo de estudio. Es preocupante la baja frecuencia de referencias al lugar en donde se encuentran depositadas las muestras o los individuos que fueron objeto de estudio. Por otra parte, los metadatos de segundo nivel presentan información desigual e incompleta.

En este trabajo se ha tomado la región de PA como caso de estudio. Sin embargo, entendemos que la problemática en torno al intercambio de datos excede los límites regionales. Este análisis es una primera aproximación que contribuye a incentivar el debate dentro de la disciplina de un tema de fundamental importancia, independientemente de la región de estudio. Si la idea de nuestra comunidad científica es compartir, entonces es necesario comenzar a generar las bases para que ello pueda ocurrir. 
Pensar globalmente y actuar localmente resume los desafíos que implican la implementación e incorporación de cambios como el "data sharing". El involucramiento y los espacios de diálogo tanto para desarrollar metodologías consensuadas (estandarizadas) como para promover formas de intercambio que sean respetuosas del trabajo de cada uno de los investigadores, puede ser un camino.

Sería interesante para continuar profundizando en el conocimiento previo al intercambio de datos en sí mismo y antes de su implementación, la realización de una encuesta a la comunidad bioantropológica de la región, para saber cuál es la predisposición y voluntad de estos ante esta nueva corriente. Poniendo la vista en la implementación de plataformas que sean de utilidad a la comunidad bioantropológica de PA.

\section{Agradecimientos}

Necochea, 14 de junio de 2014

Los autores queremos agradecer encarecidamente a todos aquellos que nos ayudaron aportándonos los artículos a los cuales no conseguimos acceso. Este trabajo fue parcialmente financiado por los proyectos PICT 0575, PICT 01520, PIP 5576, PIP/CONICET 112-200801-00996, UNPA 29/A206-1, 29/A302, 05/F723 SeCyT-UNC y UBACyT 20020090200015.

\section{Biliografía}

Ambrose, S. H. (1990). Preparation and characterization of bone and tooth collagen for isotopic analysis. Journal of Archaeological Science, 17 (4), 431-451.

Ambrose, S. H. (1993). Isotopic analysis of paleodiets: methodological and interpretive considerations. Pp. 59-130.

Azberger, P., Schroeder, P., Beaulieu, A., Bowker, G., Casey, K., Laaksonen, L., ... Wouters, P. (2004). Promoting Access to Public Research Data Scientific, Economic and Social Development. Data Science Journal, 3, 135-152.

Borgman, C. L. (2012). Advances in Information Science. The Conundrum of Sharing Research Data. Journal of the American Society for Information Science and Technology, 63(6), 10591078.

Borgman, C. L., Wallis, J. C., \& Mayernik, M. S. (2012). Who's Got the Data? Interdependencies in Science and Technology Collaborations. Computer Supported Cooperative Work, 21, 485-523.

Boulton, G. (2012). Open your minds and share your results. Nature, 486, 441.

Boulton, G., Campbell, P., Collins, B., Elias, P., Wendy Hall, D., Laurie, G., ... Walport, M. (2012). Science as an open enterprise. The Royal Society, London.

Buikstra, J.E., \& Ubelaker, D.H., (1994). Standards for Data Collection from Human Skeletal Remains. Arkansas Archaeological Survey Research Series No.44, Arkansas.
Carnese, F. R., \& Pucciarelli, H. M. (2007). Investigaciones Antropobiológicas en Argentina, desde la década de 1930 hasta la actualidad. Relaciones de la Sociedad Argentina de Antropología, XXXII, 243-280.

Chavan, V., \& Penev, L. (2011). The data paper: a mechanism to incentivize data publishing in biodiversity science. BMC Bioinformatics 12 Suppl 15: S2.

Contreras, J. L. (2010). Data Sharing, Latency variables, and Science Commons. Berkeley Technology Law Journal, 25(4), 1601-1672.

Coplen, T. B., \& Qi, H. (2009). Quality assurance and quality control in light stable isotope laboratories: A case study of Rio Grande, Texas, water samples. Isotopes in Environmental and Health Studies, 45(2), 126-134.

Coplen, T. B. (2011) Guidelines and recommended terms for expression of stable sotope ratio and gas ratio measurement results. Rapid Communications in Mass Spectrometry, 25(17), 2538-2560.

Delson, E., Harcourt-Smith, W. E. H., Frost, S., \& Norris, C. A. (2007). Databases, Data Access, and Data Sharing in Paleoanthropology: First Steps. Evolutionary Anthropology, 16, 161-163.

Destro Bisol, G., Capocasa, M., Anagnostou, P., \& Greco, P. (2013). Opening Science to Society, a new initiative of the Istututo Italiano de Antropologia. Journal of Anthropological Sciences, 91, 233-235.

Destro Bisol, G., Anagnostou, P., Capocasa, M., Bencivelli, S., Cerroni, A., Contreras, J., ... Boulton, J. (2014a). Perspectives on open science and scientific data sharing: an interdisciplinary workshop. Journal of Anthropological Sciences, 92, 1-22.

Destro Bisol, G., Anagnostou, P., \& Capocasa. M. (2014b). Cooperazione e condivisiones nella ricerca scientifica. Open Science/Open data, 1-15.

Destro Bisol, G. D., Anagnostou, P., Bruner, E., Capocasa, M., Canali, S., Danubio, M. E., ... Vona, G. (2014c). Open data, Science and Society: launching Oasis, the flagship initiative of the Istituto Italiano de Antropologia. Journal of Anthropological Science, 92: I-IV.

Elton, S., \& Cardini, A. (2008). Anthropology from the desk? The challenges of the emerging era of data sharing. Journal of Anthropological Sciences, 86, 209-212.

Eysenbach, G. (2006). Citation advantage of open access articles. PLOS Biol 4(5), e157.

Faniel, I. M., \& Zimmerman, A. (2011). Beyond the Data Deluge: A Research Agenda for Large-Scale Data Sharing and Reuse. The International Journal of Digital Curation, 1 (6), 58-69.

Frank, R. D., Yakel, E., \& Faniel, I. M. (2015). Destruction/ reconstruction: preservation of archaeological and zoological research data. Archival Science.

Fry, B. (2006). Stable Isotope Ecology. New York: Springer. 
García, R. (2006). Sistemas complejos. Conceptos, método y fundamentación epistemológica de la investigación interdisciplinaria. Barcelona, Editorial Gedisa.

González, P. N., \& Beguelín, M. (2013). Bases públicas de datos morfométricos en antropología biológica: nuevas prácticas, nuevos desafíos. Revista del Museo de Antropología, 6, 73-78.

González-José, R., García Moro, C., Dahinten, S., \& Hernandez, M. (2002). Origin of Fueguian-Patagonians: An Approach to Population History and Structure Using R Matrix and Matrix permutation Methods. American Journal of Human Biology, $14,308-320$.

González-José, R., Botolini, M. C., Santos, F. R., \& Bonatto, S. L. (2008). The Peopling of America: Craneofacial Shape Variation on a Continental Scale and its Interpretation From an Interdisciplinary View. American Journal of Physical Anthrpology, 137, 175-187.

Guichón, R. A., \& Figuerero Torres, M. J. (1991). Biología Humana y Arqueología: Propuestas y Problemas teórico-metodológicos. Artículo publicado en Shincal 3 (Tomo 2), III Congreso Nacional de Arqueología Argentina. 129-133.

Guichón, R. A. (2002). Biological Anthropology of Southern Patagonia. In C. Briones \& J. L. Lanata (Eds.), Archaeological and Anthropological Perspectives on the Native Peoples of Pampa, Patagonia, and Tierra del Fuego to the Nineteenth Century (pp. 13-29). Greenword, Pub. Co. C.T. USA.

Hendriks, P. (1999). Why Share Knowledge? The Influence of ICT on the Motivation for Knowledge Sharing. Knowledge and Process Management, 6(2), 91-100.

Holdren, J. P. (2013). Memorandum for the Heads of Executive Departments and Agencies. Office of Science and Technology Policy, Executive Office of the President, Washington, D.C.

Kansa, E. C., \& Kansa, S. W. (2013). We All Know That a 14 Is a Sheep: Data Publication and Professionalism in Archaeological Communication. Journal of Eastern Mediterranean Archaeology and Heritage Studies, 1(1), 88-97.

Kullmer, O. (2008). Benefits and risks in virtual anthropology. Journal of Anthropological Science, 86, 205-207.

Lalueza, C., Pérez-Pérez, A., Prats, E., Moreno, F., Pons, J., \& Turbón, D. (1993-94). Ausencia de la delección de 9bp COII/ tRNAlys dn aborígenes de Fuego-Patagonia mediante análisis de DNA antiguo. Anales del Instituto de la Patagonia, serie Ciencias Humanas, 22, 181-191.

Ley 26899. Honorable Congreso de la Nación Argentina. Boletín Oficial del 09 de diciembre de 2013, número: 32781, página: 3. http://repositorios.mincyt.gob.ar/recursos.php

Manzi, L. M. (2001). Territorialidad y movilidad en grupos cazadores-recolectores Selk'nam: un acercamiento a partir del pasado etnográfico. Artículo presentado en las Actas del XIII Congreso Nacional de Arqueología Argentina, Córdoba. Tomo I: 11-31.

MacMillan, D. (2014). Data Sharing and Discovery: What Libraries Need to Know. The Journal of Academic Librarianship, 40, 541-549.
Mendonça de Souza, S. M. F., \& Guichón, R. A. (2012). Paleopathology in Argentina and Brazil. In J. Buikstra \& C. Roberts (Eds.), The Global History of Paleopathology (the "Work"). New York, Oxford University.

Milia, N., Congiu, A., Anagnostou, P., Montinaro, F., Capocasa, M., Sanna, E., \& Destro Visol, G. (2012) Mine, Yours, Ours? Sharing Data on Human Genetic Variation. PLOS ONE 7(6), e37552.

Najmanovich, D. (2007). El desafío de la Complejidad: Redes, cartografías dinámicas y mundos implicados. Utopía y Praxis Latinoamericana: revista Internacional de Filosofía Iberoamericana y Teoría Social, 12 (38), 71-82.

Nelson, B. (2009). Empty archives. Nature, 461, 160-163.

National Institutes of Health (NIH) (2003). NIH Data Sharing Policy and Implementation Guidance. http://grants.nih.gov/ grants/policy/data_sharing/data_sharing_guidance.htm

Organization for Economic Cooperation and Development (OECD) (2007). OECD Principles and Guidelines for Access to Research Data from Public Funding. http://www.oecd.org/sti/ sci-tech/38500813.pdf

Pessenda, L. C. R. (1998). Laboratório de C-14. Técnicas e Aplicações. Série Didática, 57.

Piwowar, H. A., Day, R. S., Fridsma, D. B. (2007) Sharing Detailed Research Data Is Associated with Increased Citation Rate. PLOS ONE, 2(3), e308.

Pinowar, H. A., Becich, M. J., Bilofsky, H., Crowley, R. S., \& caBIG Data Sharing and Intellectual Capital Workspace (2008). Towards a data sharing culture: Recommendations for leadership from academic health centers. PLoS Med, 5(9), e183.

Pinowar, H. A. (2010). Foundational studies for measuring the impact, prevalence, and patterns of publicly sharing biomedical research data. PhD School of Medicine, University of Pittsburg.

Pinowar, H. A. (2011) Who Shares? Who Doesn't? Factors Associated with Openly Archiving Raw Research Data. PLOS ONE, 6(7), e18657.

R Development Core Team. R: A Language and Environment for Statistical Computing. Vienna, Austria: R Foundation for Statistical Computing (2008). http://www.R-project.org.

Reed, D., Barr, W.A., McPherron, S. P., Bobe, R., Geraads, D., Wynn, J. G., \& Alemseged, Z. (2015). Digital Data Collection in Paleoanthropology. Evolutionary Anthropology, 24, 238-249.

Schoefield, P. N., Bubela, T., Weaver, T., Portilla, L., Brown, S. D., Hancock, J. M., ... Rosenthal, N. (2009). Post-publication sharing of data and tools. Nature, 461, 171-173.

Smith, V. S. (2009). Data publication: towards a database of everything. BMC Research Notes, 2, 113

Summer, T. A., \& Riddle, A. T. R. (2009). Remote Anthropology: Reconciling Research Priorities with Digital Data Sharing. Journal of Antrhopological Sciences, 89, 1-3.

Tenopir, C., Allard, S., Douglass, K., Aydinoglu, A. U., Wu, 
L., Read, E., ... Frame, M. (2011) Data Sharing by Scientists: Practices and Perceptions. PLOS ONE, 6(6), e21101.

Torres-Salinas, D. (2010). Compartir datos (data sharing) en ciencia: contexto de una oportunidad. Anuario ThinkEPI, 258261

Torres-Salinas, D., Robinson-García, N., \& Cabezas-Clavijo, A. (2012). Compartir datos de instigación en ciencia: introducción al data sharing. El profesional de la información, 21(2), 173184.

Uhlir, P., \& Schröeder, P. (2007). Open Data for Global Science. Data Science Journal, 6, 189-222.

Yesner, D., Figuerero Torres, M., Guichón, R., \& Borrero, L. A. (1991). Análisis de isótopos estables en esqueletos humanos: confirmación de patrones de subsistencia etnográficos para Tierra del Fuego. Artículo presentado en Shincal 3 (Tomo2), III Congreso Nacional de Arqueología Argentina, 182-191.

\section{Información suplementaria}

\section{Anexo 1}

\section{International Journal of Osteoarchaeology}

Borrero L., Barberena, R., Franco, N., Charlin J., \& Tykot R. H. (2009). Isotopes and Rocks: Geographical Organization of Southern Patagonian Hunter-Gatherers. International Journal of Osteoarchaeology, 19, 309-327.

Macchiarelli, R., Bondioli. A., Mazurier, A., Merceron, G., \& Piana, E. L. (2006). The oldest human remains from the Beagle Channel region, Tierra del Fuego. International Journal of Osteoarchaeology, 16: 15-28.

Suby, J. A., \& Guichón, R. A. (2009). Diet, Nutrition and Femoral Robusticity of Hunter-Gatherers in Southern Patagonia: Experiences and Perspectives. International Journal of Osteoarchaeology, 19, 328-336.

Tessone A., Zangrando, A. F., Barrientos G., Goñi R., Panarello P., \& Cagnoni M. (2009). Stable isotope studies in the Salitroso Lake Basin (southern Patagonia, Argentina): assessing diet of Late Holocene hunter-gatherers. International Journal of Osteoarchaeology, 19(2), 297-308.

\section{American Journal of Physical Anthropology}

Bernal, V., González, P. N., Pérez, S. I., Sardi, M. L., \& Pucciarelli H. M. (2010). Spatial patterns and evolutionary processes in southern South America: a study of dental morphometric variation. American Journal of Physical Anthropology, 142, 95-104.

García-Bour, J., Pérez-Pérez, A., Álvarez, S., Fernández, E., López-Parra, A. M., \& Arroyo-Prado, E. (2004). Early Population Differentiation in Extinct Aborigines from Tierra del FuegoPatagonia: Ancient mtDNA Sequences and Y-Chromosome STR Characterization. American Journal of Physical Anthropology, $123,361-370$.

González, P. N., Pérez I. S., \& Bernal, V. (2010). Ontogeny of Robusticity of Craniofacial Traits in Modern Humans: A Study of Soth American Populations. American Journal of Physical Anthropology, 142, 367-379.

González José, R., Dahinten S. L., Luis M. A., Hernández, M., \& Pucciarelli, H. M. (2001). Craniometric variation and the settlement of the Americas: Testing hypothesis by means of $\mathrm{R}$ matrix and matrix correlation analysis. American Journal of Physical Anthropology, 116, 154-165.

González José, R, Brotolini, M. C., Santos, F. R., \& Bonatto, S. L. (2008). The peopling of América: Craniofacial shape variation on a continental scale and its interpretation from a interdisciplinary view. American Journal of Physical Anthropology, 137, 175-187.

Hernández, M., Lalueza, C., \& García-Moro, C. (1997a). Brief communication: Stature estimation in extinct Aónikenk and the myth of patagonian gigantism. American Journal of Physical Anthropology, 105, 545-551.

Hernández, M., Lalueza, C., \& García-Moro, C. (1997b). Fueguian cranial morphology: The adaptation to a cold, harsh environment. American Journal of Physical Anthropology, $103,103-107$.

Sardi, M., Ramírez-Rozzi, F., Dahinten, S. L., \& Pucciarelli, H. M. (2005). South Amerindian craniofacial morphology: diversity and implications for Amerindian evolution. International Journal of Osteoarchaeology, 128, 747-756.

\section{Revista Argentina de Antropología Biológica}

Bastida, R., Quse, V., \& Guichón, R. A. (2011). La tuberculosis en grupos de cazadores recolectores de Patagonia y Tierra del Fuego: nuevas alternativas de contagio a través de la fauna silvestre. Revista Argentina de Antropología Biológica, 13(1), 83-95.

Beguelín, M., \& González, P. N. (2008). Estimación del sexo en poblaciones del sur de Sudamérica mediante funciones discriminantes para el fémur. Revista Argentina de Antropología Biológica, 10(2), 55-70.

García-Laborde, P., Suby, J. A., Guichón, R. A., \& Casali, R. (2010). El antiguo Cementerio de la Misión de Río Grande, Tierra del Fuego. Primeros resultados sobre patologías nutricionalesmetabólicas e infecciosas. Revista Argentina de Antropología Biológica, 12(1), 57-70.

Guichón, R. A., Borrero, L. A., Prieto, A., Cárdenas, P., \& Tykot, R. (2001). Nuevas determinaciones de isótopos estables para Tierra del Fuego. Revista Argentina de Antropología Biológica, 3(1): 113-126.

Pérez, S. I. (2006). Influencia de la deformación artificial del cráneo sobre la morfología facial: implicancias para los estudios de relaciones evolutivas inter-poblacionales. Revista Argentina de Antropología Biológica, 8(1), 37-55.

Schinder, G., Guichón, R. A., Comparatore, V., \& Burry, S. (2003). Inferencias paleodietarias mediante isótopos estables en restos óseos humanos provenientes de Tierra del Fuego, Argentina. Revista Argentina de Antropología Biológica, 5(2), 15-31.

Suby, J. A., Guichón, R. A., \& Zangrando, A. F. (2009). El registro biológico humano de la costa meridional de Santa Cruz. Revista 
Argentina de Antropología Biológica, 11(1), 109-124.

Tessone, A., Zangrando, A. F., Valencio, S., \& Panarello, P. (2003). Isótopos Estables del Carbono en restos óseos humanos de la región del Canal de Beagle, Isla Grande de Tierra del Fuego. Revista Argentina de Antropología Biológica, 5(2), 33-43.

Intersecciones en Antropología

Beguelín, M., \& Barrientos, G. (2006). Variación morfométrica postcraneal en muestras tardías de restos humanos de Patagonia: una aproximación biogeográfica. Intersecciones en Antropología, 7, 49-62.

Beguelín, M., Lotto, F. P., \& González, P. N. (2011). Estimación del sexo en cazadores-recolectores de Sudamérica a partir de variables métricas del húmero. Intersecciones en Antropología, $12,61-68$.

García Guraieb S. (2006). Salud y enfermedad en cazadoresrecolectores del Holoceno tardío en la cuenca del lago Salitroso (Santa Cruz). Intersecciones en Antropología, 7, 37-48.

L'Heureux, G. L., Guichón, R. A., Barberena, R., \& Borrero, L. A. (2003). Durmiendo bajo el faro. Estudio de un entierro humano en Cabo Vírgenes (C.V.17), provincia de Santa Cruz, República Argentina. Intersecciones en Antropología, 4, 87-97.

L'Hereux, G., \& Barberena, R. (2008). Evidencias bioarqueológicas en Patagonia meridional: el sitio Orejas de Burro 1 (Pali Aike, provincia de Santa Cruz). Intersecciones en Antropología, 9, $11-24$.

Santiago, F. (2007). Análisis tafonómico en La Arcillosa 2, Tierra del Fuego, Argentina. Intersecciones en Antropología, 8, 15-26.

Santiago, F., Salemme, M., Suby, J. A., \& Guichón, R.A. (2011). Restos óseos humanos en el norte de Tierra del Fuego. Aspectos contextuales, dietarios y paleopatológicos. Intersecciones en Antropología, 12, 147-162.

Suby, J. A., \& Guichón, R. A. (2004). Densidad ósea y Frecuencias de hallazgos en restos humanos en el Norte de Tierra del Fuego. Análisis exploratorio. Intersecciones en Antropología, 5, 95-104.

Suby, J. A. (2006). Estudio metodológico-comparativo de densidad mineral ósea de restos humanos por Absorciometría Fotónica (DXA). Intersecciones en Antropología, 7, 277-285.

Suby, J. A., Costantino, S., Capiel, C., Lucarini, M. M., \& Etchapare, E. (2013). Exploraciones de la densidad mineral ósea y osteopenia en poblaciones humanas antiguas de Patagonia austral. Intersecciones en Antropología, 14: 433-445.

\section{Magallania}

Aguilera, N., \& Grendi, P. (1976). Hallazgo de un chenke Proto Aonikenk en Juni Aike, Magallanes. Anales del Instituto de la Patagonia, serie Ciencias Humanas, 24. 163-175.

Alfonso-Durruty, M. P., Calás, E., Morello, F. (2011). Análisis Bioantropológico de un enterramiento humano del Holoceno Tardío en Cabo Nose, Tierra del Fuego, Chile. Magallania, 39(1), 147-162.

Álvarez, M., Vázquez, M., \& Piana, E. (2008). Prácticas mortuorias entre los cazadores-recolectores del canal Beagle: el caso de Shamakush Entierro- Magallania, 38, 107-124.

Aspillaga, E. C., \& Ocampo, C. (1996). Restos óseos humanos de la Isla Karukinka (Seno Almirantazgo, Tierra del Fuego) Informe Preliminar. Anales del Instituto de la Patagonia, serie Ciencias Humanas, 24, 163-175.

Aspillaga, E. C., Ocampo, C., \& Rivas, P. H. (1999). Restos óseos humanos de contextos arqueológicos del área de isla Navarino: indicadores de estilo de vida en indígena canoeros. Anales del Instituto de la Patagonia, serie Ciencias Humanas, 27, 123.135.

Aspillaga, E. C., Castro, M., Rodríguez, M., \& Ocampo, C. (2006). Paleopatología y estilo de vida: el ejemplo de los Chonos. Magallania, 34(1), 77-85.

Bird, J. (1983). Enterratorios paleoindios con cremación en las cuevas de Palli-Aike y Cerro Sota en Chile meridional. Anales del Instituto de la Patagonia, serie Ciencias Humanas, 14. 55-65.

Borrero, L. A., Guichón, R. A., Tykot, R., Kelly, J., Prieto, A, \& Cardenas, P. (2001). Dieta a partir de Isótopos Estables en restos óseos humanos de Patagonia Austral. Estado actual y perspectivas. Anales del Instituto de la Patagonia, serie Ciencias Humanas, 29, 119-127.

Cocilovo, J. A., \& Guichón, G. A. (1985-86). Propuesta para el estudio de las poblaciones aborígenes del extremo austral de Patagonia. Anales del Instituto de la Patagonia, serie Ciencias Humanas, 16, 111-123.

Constantinescu, F. C. (1997). Hombres y mujeres del cerro Los Onas: presentes, ausentes ... los relatos de sus huesos. Anales del Instituto de la Patagonia, serie Ciencias Humanas, 25, 59-74.

Constantinescu, F. C. (1999). Evidencias bioantropológicas para modos de vida cazador recolector terrestre y marítimo en los restos humanos óseos de Tierra del Fuego. Anales del Instituto de la Patagonia, serie Ciencias Humanas, 27, 137-174.

Constantinescu, F. C. (2001). Canal Maule y Punta Santa Ana: El habitus cazador. Anales del Instituto de la Patagonia, serie Ciencias Humanas, 29, 163-181.

Constantinescu, F. C. (2003). Obsidiana verde incrustada en un cráneo Aónikenk. Magallania, 31, 149-153.

Guichón, R. A., \& Santini Araujo, E. (1987). Restos óseos humanos de Isla Englefield, Magallanes (Chile). Anales del Instituto de la Patagonia, serie Ciencias Humanas, 17, 113-117.

Guichón, R. A. (1992). Informe preliminar sobre el enterratorio con cremación del sitio Marazzi. Anales del Instituto de la Patagonia, serie Ciencias Humanas, 21. 109-112.

Guichón, R. A., Barberena, R., \& Borrero, L. A. (2001). ¿Dónde y cómo aparecen los restos óseos humanos en Patagonia austral? Anales del Instituto de la Patagonia, serie Ciencias Humanas, 29, 103-118.

Guichón, R. A., \& Suby, J. A. (2011). Estudio bioarqueológico de los restos óseos humanos recuperados por Anne Chapman en Caleta Falsa, Tierra del Fuego. Magallania, 39(1), 163-177. 
Guichón, R. A., \& Suby, J. A. (2006). La colección del Museo de Historia Natural de Historia Natural de Londres correspondiente a restos óseos humanos de Patagonia Austral. Magallania, $34(2), 47-56$.

González José, R., Martínez-Abadías, N., Van der Molen, S., García Moro, C., Dahinten, S. L., \& Hernández, M. (2004). Hipótesis del poblamiento de Tierra del Fuego-Patagonia a partir del análisis genético-poblacional de la variación craniofacial. Magallania, 32, 79-98.

Hernández, M. (1992). Morfología craneal de las etnias de la Tierra del Fuego: diferencias sexuales e intergrupales. Anales del Instituto de la Patagonia, serie Ciencias Humanas, 21, 81-98.

Lalueza, C., Pérez-Pérez, A., Prats, E., Moreno, F., Pons, J., \& Turbón, D. (1993-94). Ausencia de la delección de 9bp COII/ tRNAlys dn aborígenes de Fuego-Patagonia mediante análisis de DNA antiguo. Anales del Instituto de la Patagonia, serie Ciencias Humanas, 22, 181-191.

Lalueza, C., Pérez-Pérez, A., Prats, E., \& Turbón, D. (1995). Linajes mitocondriales de los aborígenes de Tierra del Fuego y Patagonia. Anales del Instituto de la Patagonia, serie Ciencias Humanas, 23, 75-86.

Lalueza, C., Hernández, M., \& García-Moro, C. (1997). La morfología facial de las poblaciones fueguinas: ¿Un reflejo de una adaptación al frío? Anales del Instituto de la Patagonia, serie Ciencias Humanas, 25, 45-58.

Legoupil, D. (1987). Un recién nacido descubierto en la isla Englefield (seno de Otway, Magallanes). Anales del Instituto de la Patagonia, serie Ciencias Humanas, 17, 109-111.

Legoupil, D., \& Prieto, A. (1991). Sepultura de niños canoeros en un abrigo pintado en Ultima Esperanza, Chile. Anales del Instituto de la Patagonia, serie Ciencias Humanas, 20, 133-138.

Legoupil, D. (1993-94). El archipiélago del Cabo de Hornos y la costa sur de la Isla Navarino: poblamiento y modelos económicos. Anales del Instituto de la Patagonia, serie Ciencias Humanas, 22, 101-122.

Legoupil, D., \& Sellier. P. (2004). La sepultura de la cueva Ayayema (isla Madre de Dios, Archipiélagos Occidentales de Patagonia). Magallania, 32, 114-124.

Legoupil, D., Prieto, A., \& Sellier, P. (2004). La cueva de los niños (Seno Última Esperanza): nuevos hallazgos. Magallania, $32,225-227$.

L'Heureux, G. L., \& Amorosi, T. (2009). En entierro 2 del sitio Cañadón Leona 5 (Región de Magallanes, Chile). Viejos huesos, nuevos datos. Magallania, 37(2), 41-55.

L'Heureux, G. L., \& Amorosi, T. (2010). El entierro del sitio Cerro Sota (Magallanes, Chile) a más de setenta años de su excavación. Magallania, 38(2), 133-149.

Martin, F. M. (2002). La marca del zorro. Cerro Johny, un caso arqeuológico de carroñeo sobre un esqueleto humano. Anales del Instituto de la Patagonia, serie Ciencias Humanas, 30, 133-146.

Martin, F. M., Barberena, R., \& Guichón, R. A. (2004). Erosión y huesos humanos. El caso de la localidad de Chorrillos, Tierra del Fuego. Magallania, 32, 125-142.

Martinic, M. (1976). Hallazgo y excavación de una tumba aonikenk en Cerro Johny (Brazo Norte). Anales del Instituto de la Patagonia, serie Ciencias Humanas, 7, 87-94.

Martinic, M. (1989-90). El genocidio Selk'nam: nuevos antecedentes. Anales del Instituto de la Patagonia, serie Ciencias Humanas, 19, 23-28.

Massone M. A. (1984). Los paraderos tehuelches y trototehuelches en la costa del Estrecho de Magallanes (Una aproximación teórica y metodológica). Anales del Instituto de la Patagonia, serie Ciencias Humanas, 15, 27-42.

Massone, M. A., Prieto, A., \& Cárdenas, P. (1985-86). Contexto arqueológico de un enterratorio Tehuelche excavado en la localidad de San Gregorio, Magallanes. Anales del Instituto de la Patagonia, serie Ciencias Humanas, 16, 95-101.

Massone M. A. (1986). Hombre temprano y Paleoambiente en la región de Magallanes: Evaluación crítica y perspectivas. Anales del Instituto de la Patagonia, serie Ciencias Humanas, 24, 81-98.

Moraga, M., de Saint Pierre, M., Torres, F., \& Ríos, J. (2010). Vínculos de parentesco por vía materna entre los últimos descendientes de la etnia Kawésqar y algunos entierros en los canales patagónicos: evidencia desde el estudio de linajes mitocondriales. Magallania, 38(2), 103-114.

Ocampo, C., Rivas, P., \& Aspillaga. E. (2000). Chenque en Bahía Felipe, Costa Noroccidental de Tierra del Fuego. Anales del Instituto de la Patagonia, serie Ciencias Humanas, 28, 215-223.

Ortiz-Troncoso O. (1973). Artefactos de silex de una tumba de Morro Philippi, Valle medio del río Gallegos. Anales del Instituto de la Patagonia, serie Ciencias Humanas, 4, 131-139.

Ortiz-Troncoso O. (1975). Los yacimientos de Punta Santa Ana y Bahía Buena (Patagonia Austral). Excavaciones y fechados radiocarbónicos. Anales del Instituto de la Patagonia, serie Ciencias Humanas, 6(1-2), 93-122.

Panarello, H., Zangrando, A. F., Tessone, A., Kozameh, L. F., \& Testa, N. (2006). Análisis comparativo de paleodietas humanas entre la región del Canal Beagle y Península Mitre: perspectivas desde los Isótopos Estables. Magallania, 34(2), 37-46.

Pearson, O. M., \& Millones, M. (2005). Rasgos esqueletales de adaptación al clima y a la actividad entre los habitantes aborigenes de Tierra del Fuego. Magallania, 33(1), 37-50.

Pérez-Perez, A, \& Lalueza, C. (1992). Indicadores de presión ambiental en aborígenes de Fuego-Oatagonia: Un reflejo de la adaptación a un ambiente adverso. Anales del Instituto de la Patagonia, serie Ciencias Humanas, 21, 99-108.

Piana, E. L., Tessone, A., \& Zangrando, A. F. (2006). Contextos mortuorios en la región del Canal Beagle ... del hallazgo fortuito a la búsqueda sistemática. Magallania, 34(1), 103-117.

Prieto, A. (1984). Hallazgo de un colgante decorado en Morro Chico (Magallanes). Anales del Instituto de la Patagonia, serie Ciencias Humanas, 14, 59-61. 
Prieto, A. (1991). Cazadores tempranos y tardíos en Cueva lago Sofía I. Anales del Instituto de la Patagonia, serie Ciencias Humanas, 20, 75-99.

Prieto, A., \& Schidlowsky, Y. V. (1992). Enterramiento de una niña aoenikenk en laguna Sota. Anales del Instituto de la Patagonia, serie Ciencias Humanas, 21, 63-72.

Prieto, A. (1993-94). Algunos datos en torno a los enterratorios humanos de la Región continental de Magallanes. Anales del Instituto de la Patagonia, serie Ciencias Humanas, 22, 91-100.

San Román, M., Morello. F., \& Prieto, A. (2000). Cueva de los Chingues (Parque Nacional Pali Aike), Magallanes, Chile. Historia Natural y Cultural I. Anales del Instituto de la Patagonia, serie Ciencias Humanas, 28, 125-146.

San Román, M., \& Morello, F. (2001). Canal Maule: Nuevos antecedentes sobre prácticas funerarias en el Archipiélago fueguino. Anales del Instituto de la Patagonia, serie Ciencias Humanas, 29, 149-161.

Sardi, M. L., \& Puciarelli, H. M. (2000). Posición relativa de fueguinos y araucanos en un contexto intercontinental. Un estudio multivariado de craneología funcional. Anales del Instituto de la Patagonia, serie Ciencias Humanas, 28, 73-80.

Schinder, G., \& Guichón, R. A. (2003). Isótopos estables y estilo de vida en muestras óseas humanas de Tierra del Fuego. Magallania, 31, 33-44.
Sellier. P. (1999). Los restos de los dos niños de la sepultura de Última Esperanza (Magallanes, Chile): un feto anencefálico y uno nacido muerto. Anales del Instituto de la Patagonia, serie Ciencias Humanas, 22, 99-122.

Suby, J. A., Santiago, F., \& Salemme, M. (2008). Análisis Paleopatológico de los restos humanos del sitio Puesto Pescador 1 (Tierra del Fuego). Magallania, 36(1), 121-132.

Suby, J. A., Guichón, R.A., \& Senatore, M.X. (2009). Los restos óseos humanos de Nombre de Jesús, evidencias de la salud en el primer asentamiento europeo en Patagonia Austral. Magallania, $37(2), 23-40$.

Suby, J. A., \& Guichón, R.A. (2010). Los restos óseos humanos de la colección de la Misión Salesiana "La Candelaria" (Río Grande, Argentina). Magallania, 38(2), 23-40.

Suby, J. A., \& Guichón, R.A. (2014). Nódulos de Schmorl en restos humanos arqueológicos de Patagonia Austral. Magallania, 42(1), 135-147.

Tessone, A., Zangrando, A. F., Barrientos, G., Valencio, S., Panarello, P., \& Goñi, R. (2005). Isótopos estables del carbono en Patagonía Meridional: datos de la cuenca del Lago Salitroso (Provincia Santa Cruz, República Argentina). Magallania, 33(2), 21-28. 
Anexo 2

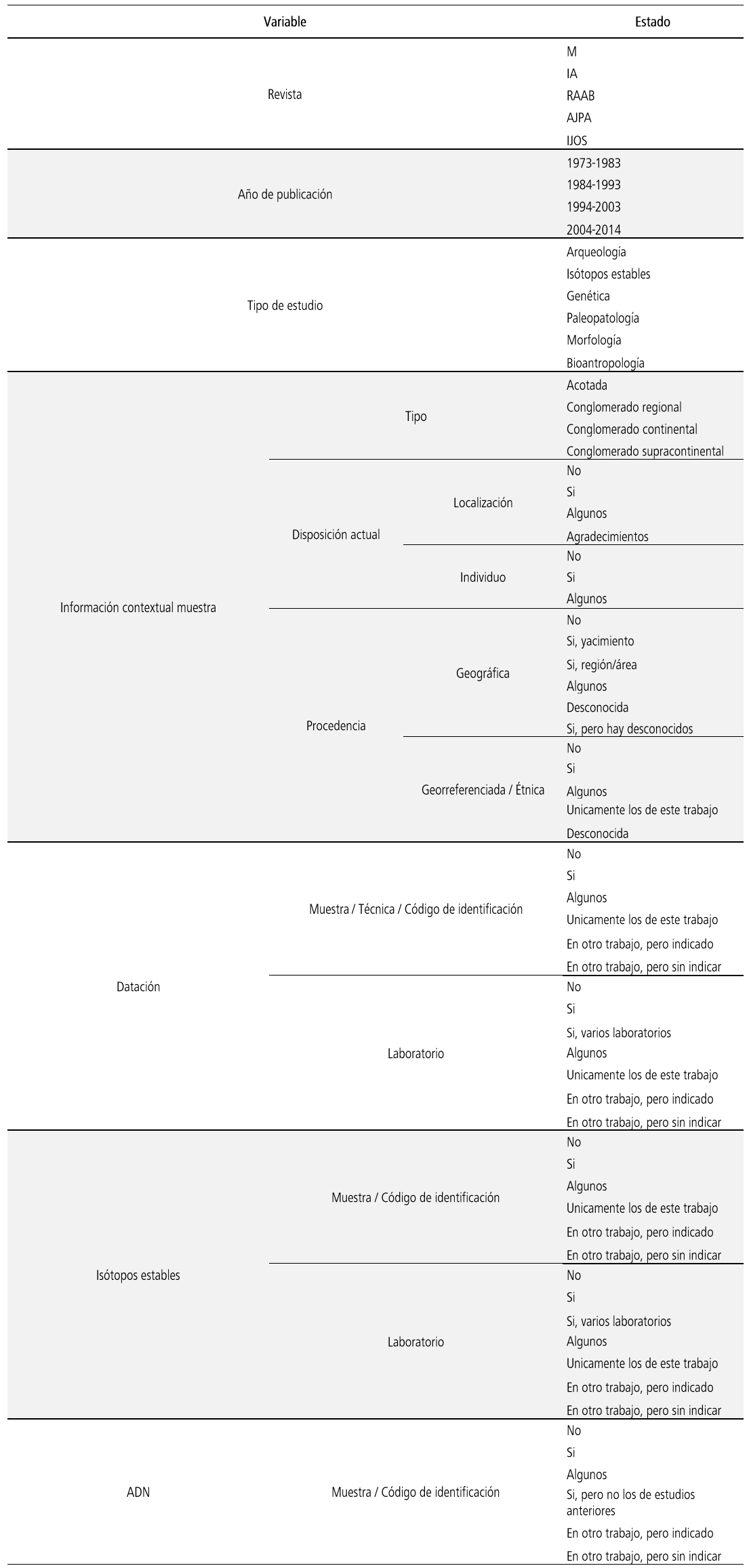

